\title{
FINITE INDEX THEOREMS FOR ITERATED GALOIS GROUPS OF CUBIC POLYNOMIALS
}

\author{
ANDREW BRIDY AND THOMAS J. TUCKER
}

\begin{abstract}
Let $K$ be a number field or a function field. Let $f \in K(x)$ be a rational function of degree $d \geq 2$, and let $\beta \in \mathbb{P}^{1}(\bar{K})$. For all $n \in \mathbb{N} \cup\{\infty\}$, the Galois groups $G_{n}(\beta)=\operatorname{Gal}\left(K\left(f^{-n}(\beta)\right) / K(\beta)\right)$ embed into $\operatorname{Aut}\left(T_{n}\right)$, the automorphism group of the $d$-ary rooted tree of level $n$. A major problem in arithmetic dynamics is the arboreal finite index problem: determining when $\left[\operatorname{Aut}\left(T_{\infty}\right): G_{\infty}(\beta)\right]<\infty$. When $f$ is a cubic polynomial and $K$ is a function field of transcendence degree 1 over an algebraic extension of $\mathbb{Q}$, we resolve this problem by proving a list of necessary and sufficient conditions for finite index. This is the first result that gives necessary and sufficient conditions for finite index, and can be seen as a dynamical analog of the Serre Open Image Theorem. When $K$ is a number field, our proof is conditional on both the $a b c$ conjecture for $K$ and Vojta's conjecture for blowups of $\mathbb{P}^{1} \times \mathbb{P}^{1}$. We also use our approach to solve some natural variants of the finite index problem for modified trees.
\end{abstract}

\section{Introduction and Statement of Results}

Let $K$ be a field. Let $f \in K(x)$ with $d=\operatorname{deg} f \geq 2$ and let $\beta \in \mathbb{P}^{1}(\bar{K})$. For $n \in \mathbb{N}$, let $K_{n}(f, \beta)=K\left(f^{-n}(\beta)\right)$ be the field obtained by adjoining the $n$th preimages of $\beta$ under $f$ to $K(\beta)$ (we declare that $K(\infty)=K$ ). Also set $K_{\infty}(f, \beta)=\bigcup_{n=1}^{\infty} K_{n}(f, \beta)$. For $n \in \mathbb{N} \cup\{\infty\}$, define $G_{n}(f, \beta)=$ $\operatorname{Gal}\left(K_{n}(f, \beta) / K(\beta)\right)$. In most of the paper, we will write $G_{n}(\beta)$ and $K_{n}(\beta)$, suppressing the dependence on $f$ if there is no ambiguity.

The group $G_{\infty}(\beta)$ embeds into $\operatorname{Aut}\left(T_{\infty}\right)$, the automorphism group of an infinite $d$-ary rooted tree $T_{\infty}$. Recently there has been much interest in the problem of determining when the index $\left[\operatorname{Aut}\left(T_{\infty}\right): G_{\infty}(\beta)\right]$ is finite. See Section 2 for background on this problem and previous results. The group $G_{\infty}(\beta)$ is the image of an arboreal Galois representation, so this finite index problem is a natural analog in arithmetic dynamics of the finite index problem for the $\ell$-adic Galois representations associated to elliptic curves, resolved by Serre's celebrated Open Image Theorem [Ser72].

We resolve the finite index problem for cubic polynomials when $K$ is a function field of transcendence degree one over an algebraic extension

2010 Mathematics Subject Classification. Primary 37P15, Secondary 11G50, 11R32, 14G25, 37P05, 37P30.

Key words and phrases. Arithmetic Dynamics, Arboreal Galois Representations, Iterated Galois Groups. 
of $\mathbb{Q}$. For such function fields, we prove an explicit list of necessary and sufficient conditions for finite index. When $K$ is a number field, our proof is conditional on both the $a b c$ conjecture and Vojta's conjecture for blowups of $\mathbb{P}^{1} \times \mathbb{P}^{1}$.

To explain our main theorem, we recall some standard terminology from arithmetic dynamics. Let $f \in K[x]$. For $n \geq 1$, let $f^{n}$ denote $f$ composed with itself $n$ times. Let $f^{0}=x$, the compositional identity. For $\alpha \in \mathbb{P}^{1}(\bar{K})$, the forward orbit of $\alpha$ under $f$ is the set $\mathcal{O}_{f}(\alpha)=\left\{f^{n}(\alpha): n \geq 1\right\}$. We say $\alpha$ is periodic for $f$ if $f^{n}(\alpha)=\alpha$ for some $n \geq 1$ and preperiodic for $f$ if $f^{n}(\alpha)=f^{m}(\alpha)$ for some $n>m \geq 1$. The point $\beta \in \mathbb{P}^{1}(\bar{K})$ is postcritical for $f$ if there is some critical point (ramification point) $\gamma$ of $f$ such that $\beta \in \mathcal{O}_{f}(\gamma)$. We say $f$ is postcritically finite or $P C F$ if only finitely many points in $\mathbb{P}^{1}(\bar{K})$ are postcritical for $f$. For $\beta \in \bar{K}$, the pair $(f, \beta)$ is stable if $f^{n}(x)-\beta$ is irreducible over $K(\beta)$ for all $n \geq 1$ and eventually stable if the number of irreducible factors of $f^{n}(x)-\beta$ over $K(\beta)$ is bounded independently of $n$ as $n \rightarrow \infty$ (stability and eventual stability can also be defined for rational functions as in JL16). If $K$ is a function field with field of constants $k$, then $f$ is isotrivial if there exists a degree 1 polynomial $\sigma \in \bar{K}(x)$ such that $\sigma \circ f \circ \sigma^{-1} \in k[x]$.

Our main theorem is as follows.

Theorem 1.1. Let $K$ be a number field or a function field of transcendence degree one over an algebraic extension of $\mathbb{Q}$. Let $f \in K[x]$ with $\operatorname{deg} f=3$ and let $\beta \in K$. If $K$ is a number field, assume the abc conjecture for $K$ and Vojta's conjecture for blowups of $\mathbb{P}^{1} \times \mathbb{P}^{1}$. If $K$ is a function field, assume that $f$ is not isotrivial.

The following are equivalent:

(1) The pair $(f, \beta)$ is eventually stable, $\beta$ is not postcritical for $f, f$ is not $P C F, f$ has distinct finite critical points $\gamma_{1}, \gamma_{2}$, and $f^{n}\left(\gamma_{1}\right) \neq f^{n}\left(\gamma_{2}\right)$ for all $n \geq 1$.

(2) The group $G_{\infty}(\beta)$ has finite index in $\operatorname{Aut}\left(T_{\infty}\right)$.

It is fairly straightforward to show that the conditions in Theorem 1.1 are necessary. The proof of their necessity (Proposition 3.3) is unconditional for both number fields and function fields, and much of the argument does not depend on the degree of $f$ or whether $f$ is a polynomial. Thus we view the failure of each condition as a natural obstruction to a finite index result. The majority of our paper is dedicated to the much harder problem of showing that, in the case of cubic polynomials, these conditions are also sufficient that is, that these are the only obstructions to finite index. It may seem that the eventual stability condition is very strong, but eventual stability is known for many explicit families of $f$ and $\beta$, and is conjectured to hold whenever $f$ is not isotrivial and $\beta$ is not periodic for $f$ (see [JL16]).

A function field $K$ of transcendence degree 1 over a field $k$ here means, as usual, a finitely generated field extension of $k$ of transcendence degree 1 such that $k$ is algebraically closed in $K$. For our theorem, we may as well 
assume that $K$ is a function field of transcendence degree 1 over $\overline{\mathbb{Q}}$ since $\operatorname{Gal}\left(K_{n}(\beta) \cdot \overline{\mathbb{Q}} / K(\beta) \cdot \overline{\mathbb{Q}}\right)$ is always a subgroup of $\operatorname{Gal}\left(K_{n}(\beta) / K(\beta)\right)$.

We also note that in the function field case, in our proof of Theorem 1.1, it does not seem to be necessary that $K$ have transcendence degree 1 over an algebraic extension of $\mathbb{Q}$. The only place where that assumption is used is in Proposition 8.1 and that result extends to function fields of transcendence dimension 1 over any field of characteristic 0 (see Remark 8.4). However, since no proof of Proposition 8.1 in this more general setting exists in the literature, we state our result only for function fields whose field of constants is an algebraic extension of $\mathbb{Q}$.

The main idea of our proof is to produce primes with specified ramification behavior in the tower of extensions $K_{n}(\beta)$ and translate this into information about the Galois groups $G_{n}(\beta)$. The basic strategy can be seen as an extension of our previous work on a Zsigmondy principle for ramification [BT18] (see also $\left[\mathrm{BIJ}^{+} 17, \mathrm{GNT}^{2}\right.$, GNT15]), which uses the Call-Silverman canonical height for dynamical systems [CS93] and the "Roth- $a b c$ " estimate as in Gra98 to show that a new prime ramifies in each $K_{n}(\beta)$ for large $n$. To extend our argument, we need stronger restrictions on ramification derived from sharper height estimates. In the number field case, these estimates come from work of Huang [Hua17] on the "dynamical gcd" problem conditional on Vojta's conjecture, Xie's [Xie15] proof of the dynamical MordellLang conjecture for $\mathbb{A}^{2}(\overline{\mathbb{Q}})$, the Medvedev-Scanlon [MS14] classification of varieties invariant under split polynomial mappings, and old work of Ritt Rit23] on polynomials that commute under composition. In the function field case, we derive the required estimates from a dynamical André-Oort theorem of Ghioca-Ye [GY16] and Favre-Gauthier [FG16], together with the Call-Silverman machinery of canonical height and specialization.

We also use our approach to solve two other finite index problems that do not seem to have been considered before. In Section 10, we replace the infinite $d$-ary tree $T_{\infty}$ with the actual tree of preimages of $\beta$ under $f$, which we call the "stunted tree" $T_{\infty}^{s}(\beta)$. The idea is that in order to identify $T_{\infty}$ with a tree of preimages of $f$, we need to count solutions to $f^{n}(x)=\beta$ with multiplicity, but if $\beta$ is periodic or postcritical for $f$, this destroys any hope of a finite index result in $\operatorname{Aut}\left(T_{\infty}\right)$ for a somewhat coarse reason. We show that passing to the stunted tree (which is a quotient tree of $T_{\infty}$ ) recovers a finite index result in many cases. With a modified notion of eventual stability, we prove Theorem 10.3 , which gives a list of necessary and sufficient conditions for finite index in $\operatorname{Aut}\left(T_{\infty}^{s}(\beta)\right.$ ) (conditional on $a b c$ and the Vojta conjecture in the case of number fields) that holds for all cubic polynomials except one exceptional family: polynomials that commute with a nontrivial Möbius transformation, which are well known to be problematic in this area. Note that this exceptional family does not need to be excluded in Theorem 1.1 . In Section 11, we simultaneously consider multiple preimage trees and prove Theorem 11.2, a similar result in this context. 
In the next section of the paper, we provide some background and definitions. Following that, in Section 3, we prove one direction of Theorem 1.1 and perform some reductions. Next, we discuss some of the issues around the notion of eventual stability in Section 4. Then, in Section 5, we summarize the height inequalities that form the backbone of the main arguments used in this paper; the inequalities here are similar those used in BT18, GNT13, GNT15. Section 6 contains some arguments from Galois theory, the most important of which (Proposition 6.7) shows that the disjointness of certain field extensions can be derived from appropriate ramification data. In the next two sections, Sections 7 and 8 , we prove the "dynamical gcd" results that allow us to deal with the fact that our polynomial has two distinct critical points; these results are what allow us to go beyond the previously studied case of unicritical polynomials. Section 9 contains the proof of Theorem 1.1, we first prove the existence of primes with certain ramification properties, in Proposition 9.1, and then apply Proposition 6.7. As described above, Sections 10 and 11 contain modifications of the tree of inverse images which allow for more general finite index results. Finally, in Section 12, we treat the case of isotrivial polynomials over function fields.

Many of the techniques used in this paper should generalize to other situations. For example, it should certainly be possible to prove a more general finite index theorem for all non-PCF cubic polynomials, assuming eventual stability over function fields and assuming eventual stability, Vojta's conjecture for blow-ups of $\mathbb{P}^{1} \times \mathbb{P}^{1}$ and the $a b c$-conjecture for number fields (here, by "finite index", we mean finite index in a natural group that may be smaller than $\left.\operatorname{Aut}\left(T_{\infty}\right)\right)$. Higher degree polynomials present additional complications, since our arguments here use the fact that any transitive subgroup of $S_{3}$ containing a transposition must be all of $S_{3}$. On the the other hand, it should be possible to use the techniques here to treat iterated Galois groups of polynomials of prime degree, since any subgroup of $S_{p}$ that contains a transposition and $p$-cycle must be all of $S_{p}$. Some new results along the lines of [FG16, GY16] would be necessary to make the proof unconditional (assuming eventual stability) over function fields. Over number fields, the required dynamical gcd results are still implied by Vojta's conjecture for blow-ups of $\mathbb{P}^{1} \times \mathbb{P}^{1}$, by work of [Hua17. Recently, Juul, Krieger, Looper, Manes, Thompson, and Walton independently discovered a similar proof of the number field case of Theorem 1.1; again, assuming $a b c$ and Vojta's conjecture. Their argument also uses Huang's [Hua17] result on Vojta's conjecture to control dynamical gcds along with methods similar to those of [BT18, GNT13] to obtain primitive prime divisors in the orbits of one of the critical points. They are working on extensions to some higher degree cases.

Finally, note the similarity of Theorem 1.1 to Conjecture 3.11 in [Jon13 for degree 2 rational functions. The main difference is that a degree 2 rational function $f$ may commute with an nontrivial Möbius transformation 
$\sigma$ such that $\sigma(\beta)=\beta$ without forcing $\beta$ to be postcritical for $f$ or $(f, \beta)$ to not be eventually stable. See [Jon13] for details.

Acknowledgements. We would like to thank Thomas Gauthier, Dragos Ghioca, Keping Huang, Rafe Jones, Nicole Looper, and Khoa Nguyen for many helpful conversations.

\section{BACKGROUND}

We give a brief introduction to arboreal Galois theory and describe some of the previous results in the area. For a comprehensive survey, see Jon13. Since we will be making repeated use of wreath products, we clarify our notation: if $G, H$ are groups and $H$ acts on a set $X$, then

$$
G \imath_{X} H=G^{X} \rtimes H,
$$

where $G^{X}$ is the direct product of $|X|$ copies of $G$ and $H$ acts on $G^{X}$ by permuting coordinates.

Let $d \geq 2$ be a fixed integer. Let $T_{n}$ denote the complete $d$-ary rooted tree of level $n$, where each non-leaf vertex has precisely $d$ child vertices. Let $T_{\infty}$ denote the complete infinite $d$-ary rooted tree, which is the direct limit of all $T_{n}$ as $n \rightarrow \infty$.

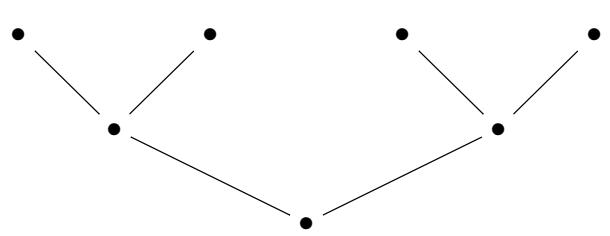

Figure $1 . T_{2}$ for $d=2$

Suppose $f \in K(x)$ with $\operatorname{deg} f=d$ and $\beta \in \mathbb{P}^{1}(\bar{K})$. The morphism $f: \mathbb{P}^{1}(\bar{K}) \rightarrow \mathbb{P}^{1}(\bar{K})$ is $d$-to- 1 away from its critical points, so the set $f^{-n}(\beta)$ generically consists of $d^{n}$ points. Let

$$
T_{n}(\beta)=\bigsqcup_{i=0}^{n} f^{-i}(\beta) .
$$

For a generic choice of $\beta$, one sees that $T_{n}(\beta)$ and $T_{n}$ are isomorphic. The root is $\beta$, the vertices at the $i$ th level are labeled by the elements of $f^{-i}(\beta)$, and the edges connect vertices labeled by $z$ and $f(z)$ for all $z \in f^{-i}(\beta)$ with $1 \leq i \leq n$. However, if $\gamma \in f^{-m}(\beta)$ is a critical point of $f$, then $f(\gamma)$ has fewer than $d$ preimages and $\left|f^{-n}(\beta)\right|<d^{n}$ for $n \geq m$. In this case, we can repair the construction as follows: we take $f^{-n}(\beta)$ to be the multiset of solutions to $f^{n}(x)=\beta$, each counted with multiplicity. Then $\left|f^{-n}(\beta)\right|=d^{n}$ for all $n$, and once again we have $T_{n}(\beta) \cong T_{n}$; see Figure 2 for examples. Note that it may be possible that some $z \in \mathbb{P}^{1}(\bar{K})$ appears in $f^{-n}(\beta)$ for two different values of $n$, which happens when $\beta$ is periodic. This 
potential repetition is why $T_{n}(\beta)$ is defined as a disjoint union, as taking a non-disjoint union would make $T_{n}(\beta)$ and $T_{n}$ non-isomorphic as trees in the case of periodic $\beta$. In Section 10 we define and study a "stunted tree" that does not keep track of repeated points in the tree due to periodic or postcritical $\beta$, with somewhat different results. Let

$$
T_{\infty}(\beta)=\bigsqcup_{i=0}^{\infty} f^{-i}(\beta)
$$

be the infinite $d$-ary rooted tree formed by taking the direct limit of all the $T_{n}(\beta)$. With our convention on counting with multiplicity, $T_{\infty}(\beta)$ and $T_{\infty}$ are isomorphic as rooted trees for every $\beta \in \mathbb{P}^{1}(\bar{K})$.

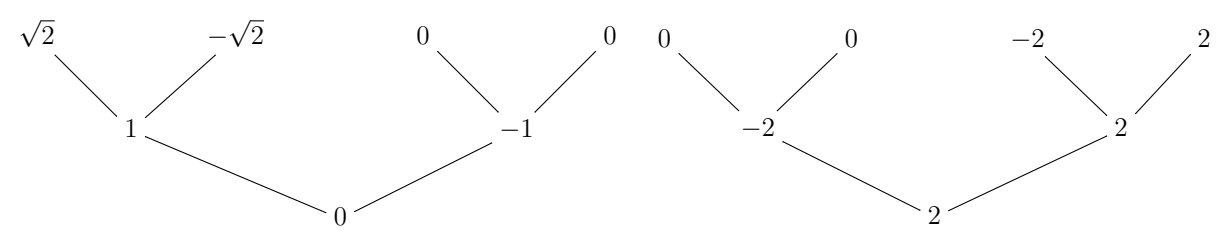

Figure 2. $T_{2}(0)$ for $x^{2}-1$ and $T_{2}(2)$ for $x^{2}-2$

As in the introduction, for $n \in \mathbb{N} \cup\{\infty\}$, set $K_{n}(\beta)=K\left(f^{-n}(\beta)\right)$ and $G_{n}(\beta)=\operatorname{Gal}\left(K_{n}(\beta) / K(\beta)\right)$. The fields $K_{n}(\beta)$ are obviously Galois extensions of $K(\beta)$. Note that $G_{\infty}(\beta)$ is isomorphic to the inverse limit of the groups $G_{n}(\beta)$ with the natural projection maps $G_{n+1}(\beta) \rightarrow G_{n}(\beta)$.

The starting point of arboreal Galois theory is the observation that, for all $n \geq 1$, the group $G_{n}(\beta)$ acts faithfully by automorphisms on $T_{n}(\beta)$. Edges in $T_{n}(\beta)$ are determined by the action of $f$ on $T_{n}(\beta) \backslash \beta$, and the action of $G_{n}(\beta)$ on $T_{n}(\beta)$ commutes with the action of $f$ because $f \in K(x)$. Thus there is an injection

$$
G_{n}(\beta) \hookrightarrow \operatorname{Aut}\left(T_{n}(\beta)\right) \cong \operatorname{Aut}\left(T_{n}\right),
$$

where $\operatorname{Aut}\left(T_{n}\right)$ is the automorphism group of $T_{n}$ as a rooted tree. It is also clear that $\operatorname{Gal}\left(K_{n}(\beta) / K_{n-1}(\beta)\right)$ injects into $\operatorname{Aut}\left(T_{n} / T_{n-1}\right)$, the subgroup of $\operatorname{Aut}\left(T_{n}\right)$ that fixes the $(n-1)$ st level of the tree.

Certainly, $\operatorname{Aut}\left(T_{1}\right) \cong S_{d}$. It is easy to show inductively that

$$
\operatorname{Aut}\left(T_{n}\right) \cong S_{d} \imath_{T_{n-1} \backslash T_{n-2}} \operatorname{Aut}\left(T_{n-1}\right)
$$

from which follows the order formula

$$
\left|\operatorname{Aut}\left(T_{n}\right)\right|=(d !)^{d^{n-1}}\left|\operatorname{Aut}\left(T_{n-1}\right)\right|=(d !)^{d^{n-1}+d^{n-2}+\cdots+1}=(d !)^{\frac{d^{n}-1}{d-1}} .
$$

Also observe that $\operatorname{Aut}\left(T_{n} / T_{n-1}\right) \cong\left(S_{d}\right)^{\left|T_{n-1} \backslash T_{n-2}\right|}$, so

$$
\left|\operatorname{Aut}\left(T_{n} / T_{n-1}\right)\right|=(d !)^{d^{n-1}}
$$


The group $\operatorname{Aut}\left(T_{n}\right)$ is often described as an "iterated wreath product"

$$
\operatorname{Aut}\left(T_{n}\right) \cong S_{d} \prec S_{d} \prec S_{d} \prec \cdots \succ S_{d}
$$

though this is somewhat misleading, as every instance of the symbol $\zeta$ in the above line implicitly refers to a group action on a distinct set.

Taking inverse limits, there is an injection $G_{\infty}(\beta) \hookrightarrow \operatorname{Aut}\left(T_{\infty}\right)$. Equivalently, we have the arboreal Galois representation

$$
\rho_{f, \beta}: \operatorname{Gal}(\bar{K} / K) \rightarrow \operatorname{Aut}\left(T_{\infty}\right),
$$

where $\rho_{f, \beta}$ sends an element of $\operatorname{Gal}(\bar{K} / K)$ to the induced automorphism of $T_{\infty}$. Then $G_{\infty}(\beta)$ is the image of $\rho_{f, \beta}$ inside $\operatorname{Aut}\left(T_{\infty}\right)$. The main problem in the field is to determine the "size" of $G_{\infty}(\beta)$, for example as in the following motivating question.

Question 2.1. Let $K, f$, and $\beta$ be as above.

(1) When is $\left[\operatorname{Aut}\left(T_{\infty}\right): G_{\infty}(\beta)\right]=1$ ?

(2) When is $\left[\operatorname{Aut}\left(T_{\infty}\right): G_{\infty}(\beta)\right]<\infty$ ?

The first part of Question 2.1 was originally studied by Odoni Odo85, Odo88, who showed that $\left[\operatorname{Aut}\left(T_{\infty}\right): G_{\infty}(\beta)\right]=1$ (i.e. $\rho_{f, \beta}$ is surjective) when $f$ is a polynomial with generic (transcendental) coefficients. More recently, Juul showed that $G_{\infty}(\beta)=\operatorname{Aut}\left(T_{\infty}\right)$ for generic rational functions Juu16. Despite the work of Odoni and Juul, it is hard to show that $G_{\infty}(\beta)=\operatorname{Aut}\left(T_{\infty}\right)$ for any particular choice of $f$ and $\beta$. Still unresolved is Odoni's conjecture Odo85, Conjecture 7.5] that for every $d \geq 2$, there exists a degree $d$ polynomial with $G_{\infty}(\beta)=\operatorname{Aut}\left(T_{\infty}\right)$ (though recent work of Looper [Loo16] gives such a polynomial in every prime degree).

The second part of Question 2.1 is perhaps more natural, because the answer is invariant under replacing $K$ by a finite extension (see Proposition 3.2. The arboreal finite index problem is a natural analog in arithmetic dynamics of the finite index problem for the $\ell$-adic Galois representations associated to elliptic curves, solved by Serre's Open Image Theorem [Ser72]. Following Serre's theorem, some obstructions to finite index can be thought of as dynamical analogs of complex multiplication.

We comment briefly on the general strategy for showing that $G_{\infty}(\beta)$ has finite or infinite index in $\operatorname{Aut}\left(T_{\infty}\right)$. Consider the natural projection map $\phi_{n}: \operatorname{Aut}\left(T_{\infty}\right) \rightarrow T_{n}$. The restriction of $\phi_{n}$ to $G_{\infty}(\beta)$ maps onto $G_{n}(\beta)$. By elementary group theory, $\left[\operatorname{Aut}\left(T_{\infty}\right): G_{\infty}(\beta)\right] \geq\left[\operatorname{Aut}\left(T_{n}\right): G_{n}(\beta)\right]$. So if $\left[\operatorname{Aut}\left(T_{n}\right): G_{n}(\beta)\right] \rightarrow \infty$ as $n \rightarrow \infty$, or equivalently if

$$
\left[\operatorname{Aut}\left(T_{n} / T_{n-1}\right): \operatorname{Gal}\left(K_{n}(\beta) / K_{n-1}(\beta)\right)\right]>1
$$

for infinitely many $n$, then $\left[\operatorname{Aut}\left(T_{\infty}\right): G_{\infty}(\beta)\right]=\infty$. On the other hand, the profinite structure of $G_{\infty}(\beta)$ implies that distinct cosets of $G_{\infty}(\beta)$ in $T_{\infty}$ must map under $\phi_{n}$ to distinct cosets of $G_{n}(\beta)$ in $T_{n}$ for some $n$. So if $\left[\operatorname{Aut}\left(T_{n}\right): G_{n}(\beta)\right]$ remains bounded as $n \rightarrow \infty$, or equivalently if

$$
\operatorname{Gal}\left(K_{n}(\beta) / K_{n-1}(\beta)\right)=\operatorname{Aut}\left(T_{n} / T_{n-1}\right) \cong\left(S_{d}\right)^{d^{n-1}}
$$


for all large $n$, then $\left[\operatorname{Aut}\left(T_{\infty}\right): G_{\infty}(\beta)\right]<\infty$.

For more on arboreal Galois representations and related topics, see for example [Cre89, Sto92, BJ07, BJ09, Jon07, Jon08, Jon15, JM14, Hin13, Hin15, HJM15, Kri13, Ing13, IS09, BIJ ${ }^{+} 17$, Pin13a, Pin13b, Pin13c. Most of the previous work is for polynomials and rational functions of degree 2 . The following are typical theorems.

Theorem 2.2. Jon08 Let $K=\mathbb{Q}$. Let $f \in \mathbb{Z}[x]$ be monic with $\operatorname{deg} f=2$. Suppose that $f$ is not PCF, $(f, 0)$ is stable, and 0 is strictly preperiodic for $f$. Then $\left[\operatorname{Aut}\left(T_{\infty}\right): G_{\infty}(0)\right]<\infty$.

Theorem 2.3. GNT13] Let $K=\mathbb{Q}$. Let $f \in \mathbb{Z}[x]$ be monic with $\operatorname{deg} f=$ 2. Suppose that $f$ is not PCF and that $(f, 0)$ is stable. Assume the abc conjecture for $\mathbb{Q}$. Then $\left[\operatorname{Aut}\left(T_{\infty}\right): G_{\infty}(0)\right]<\infty$.

Our current paper deals only with the case of cubic polynomials, but we will treat the case of quadratic polynomials in future work. It is not difficult to prove analogs of the main theorems of this paper for quadratics using our techniques here. With a bit more work, and an extension of [GKNY17, we obtain, in [BGHT17, a stronger variant of Theorem 11.2 that applies to families of inverses of images of points under several different quadratic polynomials. These results are unconditional for quadratic polynomials over function fields, and require $a b c$ and Vojta's conjecture for number fields. We note that in [DLT17] it is shown that that if $f$ is a nonisotrivial quadratic polynomial over a function field and $\beta$ is not periodic, then $(f, \beta)$ is eventually stable.

Remark 2.4. It should be noted that index is not the only measure of the size of a subgroup relative to $\operatorname{Aut}\left(T_{\infty}\right)$. Hausdorff dimension is a refined measurement of the size of an infinite index subgroup linked to interesting dynamical and arithmetic properties. See [BJ07, BJ09, BFH $\left.{ }^{+} 16\right]$.

\section{Reductions and Obstructions}

In this section we prove some reductions that will be used in the proof of Theorem 1.1. For many of our arguments, it will be convenient to make a change of variables so that our cubic polynomial $f$ is monic and the quadratic term vanishes. This may require taking a finite extension of the ground field $K$. In Proposition 3.2, we show that neither of these modifications affects the answer to the finite index question.

We begin with the following standard lemma from Galois theory, which we state without proof.

Lemma 3.1. Let $M_{1} \subset M_{2}$ be a field extension of finite degree and let $M_{1} \subseteq M_{3}$ be any field extension. Then we have

$$
\left[M_{2} \cdot M_{3}: M_{3}\right] \leq\left[M_{2}: M_{1}\right] .
$$


If $\left[M_{3}: M_{1}\right]$ is finite, we also have

$$
\left[M_{2} \cdot M_{3}: M_{3}\right] \geq \frac{\left[M_{2}: M_{1}\right]}{\left[M_{3}: M_{1}\right]} .
$$

Proposition 3.2. Let $f \in K(x)$ with $\operatorname{deg} f \geq 2$ and let $\beta \in \mathbb{P}^{1}(\bar{K})$. Let $K^{\prime}$ be any extension of $K$. For $n \in \mathbb{N} \cup\{\infty\}$, let $K_{n}^{\prime}(f, \beta)=K^{\prime}\left(f^{-n}(\beta)\right)$ and $G_{n}^{\prime}(f, \beta)=\operatorname{Gal}\left(K_{n}^{\prime}(f, \beta) / K^{\prime}(\beta)\right)$. Let $\sigma \in K(x)$ have degree one, and let $g=\sigma \circ f \circ \sigma^{-1}$. Then $\left[\operatorname{Aut}\left(T_{\infty}\right): G_{\infty}(f, \beta)\right]$ must be finite whenever $\left[\operatorname{Aut}\left(T_{\infty}\right): G_{\infty}^{\prime}(f, \beta)\right]$ is finite. Furthermore, if $\left[K^{\prime}: K\right]$ is finite, then the following are equivalent:

(1) $\left[\operatorname{Aut}\left(T_{\infty}\right): G_{\infty}(f, \beta)\right]<\infty$

(2) $\left.\left[\operatorname{Aut}\left(T_{\infty}\right): G_{\infty}^{\prime}(f, \beta)\right)\right]<\infty$

(3) $\left[\operatorname{Aut}\left(T_{\infty}\right): G_{\infty}(g, \sigma(\beta))\right]<\infty$

Proof. We have $\left|G_{n}^{\prime}(f, \beta)\right| \leq\left|G_{n}(f, \beta)\right|$ by (3.1.1), so, for any extension $K^{\prime}$ of $K$, we see that $(2)$ implies $(1)$. When $\left[K^{\prime}: K\right]$ is finite, we have

$$
\left|G_{n}^{\prime}(f, \beta)\right| \geq \frac{\left|G_{n}(f, \beta)\right|}{\left[K^{\prime}: K\right]}
$$

by (3.1.2), and so (1) implies (2) as well. Now, $\left[K^{\prime}: K(\beta)\right]$ is finite so by the same reasoning, we see that $\left[\operatorname{Aut}\left(T_{\infty}\right): \operatorname{Gal}\left(K_{\infty}(g, \sigma(\beta)) \cdot K^{\prime} / K^{\prime}(\beta)\right)\right]<\infty$ if and only if $\left[\operatorname{Aut}\left(T_{\infty}\right): G_{\infty}(g, \sigma(\beta))\right]<\infty$. For each $n$, we have $K_{n}(g, \sigma(\beta))$. $K^{\prime}=K_{n}(f, \beta) \cdot K^{\prime}$, since $g^{-n}(\sigma(\beta))=\sigma\left(f^{-n}(\beta)\right)$ and $\sigma$ is defined over $K^{\prime}$. Thus, (2) and (3) are equivalent.

Applying Proposition 3.2 and changing coordinates, we may assume in the proof of Theorem 1.1 that our polynomial $f$ is of the form

$$
f(x)=x^{3}-3 a^{2} x+b
$$

where $a, b \in K$, so that $\pm a$ are the critical points of $f$. Furthermore, as noted before, we may assume in the function field case that $K$ is a function field of transcendence degree one over $\overline{\mathbb{Q}}$.

Proposition 3.3. Let $f \in K(x)$ with $d=\operatorname{deg} f \geq 2$ and let $\beta \in K$. Suppose that any of the following holds:

(1) the map $f: \mathbb{P}^{1}(\bar{K}) \rightarrow \mathbb{P}^{1}(\bar{K})$ is $P C F$;

(2) the pair $(f, \beta)$ is not eventually stable;

(3) $\beta$ is postcritical for $f$;

(4) $f \in K[x], d \geq 3$ and $f$ has precisely one finite critical point; or

(5) $f \in K[x], d=3$ and $f$ has distinct finite critical points $\gamma_{1} \neq \gamma_{2}$ such that there is some $n \geq 1$ with $f^{n}\left(\gamma_{1}\right)=f^{n}\left(\gamma_{2}\right)$.

Then $\left[\operatorname{Aut}\left(T_{\infty}\right): G_{\infty}(\beta)\right]=\infty$.

Proof. For (1), see Theorem 3.1 in [Jon13]. For (2), see Proposition 2.2 and Proposition 3.3 in JL16.

For $(3)$, if $f^{n}(\gamma)=\beta$ for some $n$ and critical point $\gamma$, then $T_{\infty}(\beta)$ has repeated vertices at the $n$th level and above. That is, $f^{n}(x)-\beta$ has at most 
$d^{n}-1$ roots in $\bar{K}$, whence $f^{n+1}(x)-\beta$ has at most $d^{n+1}-d$ roots, and in general, $f^{n+k}(x)-\beta$ has at most $d^{n+k}-d^{k}$ roots. So

$$
\left|\operatorname{Gal}\left(K_{n+k}(\beta) / K_{n+k-1}(\beta)\right)\right| \leq\left|S_{d}\right|^{d^{n+k-1}-d^{k-1}} .
$$

$\operatorname{As}\left|\operatorname{Aut}\left(T_{n+k} / T_{n+k-1}\right)\right|=(d !)^{d^{n+k-1}}$, it follows that

$$
\left[\operatorname{Aut}\left(T_{n+k} / T_{n+k-1}\right): \operatorname{Gal}\left(K_{n+k}(\beta) / K_{n+k-1}(\beta)\right)\right] \geq(d !)^{d^{k-1}}
$$

for $k \geq 0$, and so $\left[\operatorname{Aut}\left(T_{\infty}\right): G_{\infty}(\beta)\right]=\infty$.

To prove (4), observe that if $f$ has only one finite critical point $\gamma$, then up to a change of variables (and replacing $K$ by a finite extension), $f(x)=x^{d}+c$ for some $c \in K$. So $K_{n}(\beta)$ is an extension of $K_{n-1}(\beta)$ attained by adjoining an appropriate number of $d$ th roots. Adjoining a $d$ th root of unity to $K$ if necessary, it is easy to see that $\operatorname{Gal}\left(K_{n}(\beta) / K_{n-1}(\beta)\right)$ is contained in the direct product of $d^{n-1}$ copies of $C_{d}$, the cyclic group of order $d$, whereas $\operatorname{Aut}\left(T_{n} / T_{n-1}\right)$ is the direct product of $d^{n-1}$ copies of $S_{d}$. As $d \geq 3, C_{d}$ is a proper subgroup of $S_{d}$. So $\operatorname{Gal}\left(K_{n}(\beta) / K_{n-1}(\beta)\right)<\operatorname{Aut}\left(T_{n} / T_{n-1}\right)$ for all $n$, and $G_{\infty}(\beta)$ cannot have finite index in $\operatorname{Aut}\left(T_{\infty}\right)$. See [HJM15] for more discussion of iterated Galois groups of polynomials with one finite critical point.

In the situation where there is an $n$ such that $f^{n}\left(\gamma_{1}\right)=f^{n}\left(\gamma_{2}\right)$, where $\gamma_{1}$ and $\gamma_{2}$ are not preperiodic, Grell Gre17] has given an explicit description of the profinite iterated monodromy group of $f$, following work of Pink in the quadratic case Pin13b. (If $\gamma_{1}, \gamma_{2}$ are preperiodic, then $f$ is PCF.) In particular, these groups have infinite index in $\operatorname{Aut}\left(T_{\infty}\right)$, and thus the iterated Galois groups of such $f$ must as well. This proves that $\left[\operatorname{Aut}\left(T_{\infty}\right)\right.$ : $\left.G_{\infty}(\beta)\right]=\infty$ when (5) holds.

\section{Eventual Stability}

Recall the definitions of stability and eventual stability from the introduction. We establish some facts about eventually stable rational functions that will be used later. First we recall an basic result of algebra known as Capelli's Lemma. For a proof, see [FS96, p. 490].

Lemma 4.1 (Capelli). Let $K$ be a field, $f(x), g(x) \in K[x]$, and let $\alpha \in \bar{K}$ be any root of $g(x)$. Then $g(f(x))$ is irreducible over $K$ if and only if both $g$ is irreducible over $K$ and $f(x)-\alpha$ is irreducible over $K(\alpha)$.

Proposition 4.2. Let $f \in K[x]$ and $\beta \in \bar{K}$. Suppose that the pair $(f, \beta)$ is eventually stable. Then the following hold.

(1) For all sufficiently large $n$ and any $\alpha \in f^{-n}(\beta), f(x)-\alpha$ is irreducible over $K(\alpha)$.

(2) There exists $n$ such that for any $\alpha \in f^{-n}(\beta)$, the pair $(f, \alpha)$ is stable.

(3) The point $\beta$ is not periodic for $f$. 
Proof. Let $m$ be the maximum number of irreducible factors over $K(\beta)$ of $f^{n}(x)-\beta$ for any $n \geq 1$, so that

$$
f^{k}(x)-\beta=g_{1}(x) \cdots g_{m}(x)
$$

for some $k$ and some irreducible polynomials $g_{1}, \ldots, g_{m} \in K(\beta)[x]$. Then for any $n \geq k$, we have

$$
f^{n}(x)-\beta=g_{1}\left(f^{n-k}(x)\right) \cdots g_{m}\left(f^{n-k}(x)\right),
$$

and each $g_{i}\left(f^{n-k}(x)\right)$ is irreducible over $K(\beta)$, as otherwise $m$ would not be the maximum number of irreducible factors of $f^{n}(x)-\beta$.

Let $\theta_{i}=g_{i} \circ f^{n-k}$. For any $\alpha \in f^{-n}(\beta)$, we have $f^{n}(\alpha)=\beta$ and so $\theta_{i}(\alpha)=0$ for some $i$. Writing

$$
f^{n+1}(x)-\beta=\theta_{1}(f(x)) \cdots \theta_{m}(f(x))
$$

shows that each $\theta_{i}(f(x))$ is irreducible over $K$. By Capelli's Lemma, $f(x)-\alpha$ is irreducible over $K(\alpha)$, proving (1).

Now let $n$ be large and let $\alpha \in f^{-n}(\beta)$. By (1), for any $m \geq 1$ and any $z \in f^{-m}(\alpha)$, we have $[K(z): K(f(z))]=\operatorname{deg} f$. Thus $[K(z): K(\alpha)]=$ $(\operatorname{deg} f)^{m}$. Therefore $f^{m}(x)-\alpha$ is irreducible over $K(\alpha)$. So the pair $(f, \alpha)$ is stable, proving (2).

Finally, if $\beta$ is periodic for $f$, then there are infinitely many $n$ such that $f^{n}(\beta)=\beta$. If we set $\alpha=f(\beta)$, then $\alpha \in f^{-(n-1)}(\beta)$ for each of these $n$. But then $f(x)-\alpha$ is certainly not irreducible over $K(\alpha)$, because $\beta$ is a root, contradicting (1). This proves (3).

Remark 4.3. In [JL16], Jones and Levy conjecture that the converse of part (3) of Proposition 4.2 holds for all non-isotrivial $f$. If this conjecture holds, it would somewhat clarify the list of finite index obstructions in Theorem 1.1, as the eventual stability condition could be replaced by a simpler non-periodicity condition.

\section{Height Estimates}

In this section we prove a variety of height estimates that will be used in the proof of Theorem 1.1. For background on heights, see [HS00, GNT13, BT18. We set some notation below.

If $K$ is a number field, let $\mathfrak{o}_{K}$ be its ring of integers. If $K$ is a function field, choose a prime $\mathfrak{q}$ of $K$ and set

$$
\mathfrak{o}_{K}=\left\{z \in K: v_{\mathfrak{p}}(z) \geq 0 \text { for all } \mathfrak{p} \neq \mathfrak{q}\right\} .
$$

Let $\mathfrak{p}$ be a non-archimedean prime of $K$, and let $k_{\mathfrak{p}}$ be the residue field $\mathfrak{o}_{K} / \mathfrak{p}$. If $K$ is a number field, define

$$
N_{\mathfrak{p}}=\frac{1}{[K: \mathbb{Q}]} \log \# k_{\mathfrak{p}}
$$

and if $K$ is a function field with field of constants $k$, instead define

$$
N_{\mathfrak{p}}=\left[k_{\mathfrak{p}}: k\right] \text {. }
$$


For $z \in K$, let $h(z)$ denote the logarithmic height of $z$. For $f \in K[x]$ with $\operatorname{deg} f=d \geq 2$, let $h_{f}(z)$ denote the Call-Silverman canonical height of $z$ relative to $f$ CS93], defined by

$$
h_{f}(z)=\lim _{n \rightarrow \infty} \frac{h\left(f^{n}(z)\right)}{d^{n}} .
$$

We will often write sums indexed by primes that satisfy some condition. These are taken to be primes of $\mathfrak{o}_{K}$. As an example of our indexing convention, observe that

$$
\sum_{v_{\mathfrak{p}}(z)>0} v_{\mathfrak{p}}(z) N_{\mathfrak{p}} \leq h(z)
$$

by the product formula for $K$.

We make use of the notion of good reduction of a map $f \in K(x)$ at a prime $\mathfrak{p}$, which for a polynomial

$$
f(x)=a_{d} x^{d}+a_{d-1} x^{d-1}+\cdots+a_{1} x+a_{0}
$$

means that $v_{\mathfrak{p}}\left(a_{d}\right)=0$ and $v_{\mathfrak{p}}\left(a_{i}\right) \geq 0$ for $0 \leq i \leq d-1$. See MS94 or [Sil07, Theorem 2.15] for a more careful definition that also applies to rational functions. There are only finitely many primes $\mathfrak{p}$ such that $f$ has bad reduction (that is, does not have good reduction) at $\mathfrak{p}$. The main consequence of good reduction we will use is that if $f$ has good reduction at $\mathfrak{p}$, then $f$ commutes with the reduction $\bmod \mathfrak{p} \operatorname{map} \mathbb{P}^{1}(\bar{K}) \rightarrow \mathbb{P}^{1}\left(\bar{k}_{\mathfrak{p}}\right)$. This is clear for polynomials; see [Sil07, Theorem 2.18] for a proof in general. We further say that $f$ has good separable reduction at $\mathfrak{p}$ if the reduced map $\bar{f}: \mathbb{P}^{1}\left(\bar{k}_{\mathfrak{p}}\right) \rightarrow \mathbb{P}^{1}\left(\bar{k}_{\mathfrak{p}}\right)$ is separable.

Lemma 5.1. Let $f \in K[x]$ with $d=\operatorname{deg}(f) \geq 2$. Let $\gamma, \beta \in K$ such that $h_{f}(\gamma)>0$ and $\beta \notin \mathcal{O}_{f}(\gamma)$. For any $\delta>0$, there exists a constant $B_{\delta}$ with $\frac{1}{d^{B} \delta}<\frac{\delta}{4}$ such that for any $n>B_{\delta}$, we have

$$
\sum_{m=1}^{n-B_{\delta}} \sum_{v_{\mathfrak{p}}\left(f^{m}(\gamma)-\beta\right)>0} N_{\mathfrak{p}} \leq \delta d^{n} h_{f}(\gamma) .
$$

Moreover, for all sufficiently small $\delta$, we have $B_{\delta} \leq-\frac{\log \delta}{\log d}$.

Proof. This is a variant of the inequality that underlies Proposition 5.1 of GNT13. By the product formula,

$$
\sum_{m=1}^{n-B_{\delta}} \sum_{v_{\mathfrak{p}}\left(f^{m}(\gamma)-\beta\right)>0} N_{\mathfrak{p}} \leq \sum_{m=1}^{n-B_{\delta}} h\left(f^{m}(\gamma)-\beta\right) .
$$

Recall that the canonical height $h_{f}$ satisfies $h_{f}(f(z))=d h_{f}(z)$ and that $\left|h(z)-h_{f}(z)\right|$ is uniformly bounded by a constant $C_{f}$ for all $z \in K$ CS93. Also note that $|h(z-\beta)-h(z)|$ is uniformly bounded by a constant $C_{\beta}$ depending only on $\beta$ for all $z \in K$. Choose $B_{\delta}$ such that $\frac{\delta}{4 d} \leq \frac{1}{d^{B} \delta}<\frac{\delta}{4}$ and $\frac{\delta}{2} d^{n} h_{f}(\gamma)>n\left(C_{\beta}+C_{f}\right)$ for all $n>B_{\delta}$. Note that it is always possible to 
choose such a $B_{\delta}$, as the conclusion of the Lemma holds upon replacing $\delta$ with any positive $\delta^{\prime} \leq \delta$, and $\frac{n\left(C_{\beta}+C_{f}\right)}{d^{n} h_{f}(\gamma)} \rightarrow 0$ as $n \rightarrow \infty$.

If $n>B_{\delta}$, then

$$
\begin{aligned}
\sum_{m=1}^{n-B_{\delta}} h\left(f^{m}(\gamma)-\beta\right) & \leq \sum_{m=1}^{n-B_{\delta}}\left(h\left(f^{m}(\gamma)\right)+C_{\beta}\right) \\
& \leq \sum_{m=1}^{n-B_{\delta}}\left(h_{f}\left(f^{m}(\gamma)\right)+C_{\beta}+C_{f}\right) \\
& =n\left(C_{\beta}+C_{f}\right)+\sum_{m=1}^{n-B_{\delta}} d^{m} h_{f}(\gamma) \\
& \leq n\left(C_{\beta}+C_{f}\right)+\frac{d^{n} h_{f}(\gamma)}{d^{B_{\delta}}} \sum_{r=0}^{\infty} \frac{1}{d^{r}} \\
& \leq n\left(C_{\beta}+C_{f}\right)+\frac{\delta}{2} d^{n} h_{f}(\gamma) \\
& \leq \delta d^{n} h_{f}(\gamma) .
\end{aligned}
$$

We have $\frac{\delta}{4 d} \leq \frac{1}{d^{B} \delta}$, so $\log 4 d-\log \delta \geq B_{\delta} \log d$, and

$$
B_{\delta} \leq-\frac{\log \delta}{\log d}
$$

Note that in the statements of Lemmas 5.2, 5.3, and 5.4, the chosen $\beta_{1}$ and $\beta_{2}$ in $K$ need not be distinct.

Lemma 5.2. Let $f \in K[x]$ with $d=\operatorname{deg}(f) \geq 2$. Let $\gamma \in K$ with $h_{f}(\gamma)>0$. Let $\beta_{1}, \beta_{2} \in K$ such that $\beta_{2} \notin \mathcal{O}_{f}\left(\beta_{1}\right)$. For $n>0$, let $\mathcal{X}(n)$ denote the set of primes $\mathfrak{p}$ of $\mathfrak{o}_{K}$ such that

$$
\min \left(v_{\mathfrak{p}}\left(f^{m}(\gamma)-\beta_{1}\right), v_{\mathfrak{p}}\left(f^{n}(\gamma)-\beta_{2}\right)\right)>0
$$

for some $0<m<n$. Then for any $\delta>0$, we have

$$
\sum_{\mathfrak{p} \in \mathcal{X}(n)} N_{\mathfrak{p}} \leq \delta d^{n} h_{f}(\gamma)+O_{\delta}(1)
$$

for all $n$.

Proof. For the finitely many primes $\mathfrak{p}$ such that $f$ has bad reduction at $\mathfrak{p}$, we can absorb any contribution to the sum of $N_{\mathfrak{p}}$ into $O_{\delta}(1)$, so we can assume that the primes in $\mathcal{X}(n)$ are primes of good reduction for $f$. Then if

$$
\min \left(v_{\mathfrak{p}}\left(f^{m}(\gamma)-\beta_{1}\right), v_{\mathfrak{p}}\left(f^{n}(\gamma)-\beta_{2}\right)\right)>0
$$


we have $f^{m}(\gamma) \equiv \beta_{1}(\bmod \mathfrak{p})$ and $f^{n}(\gamma) \equiv \beta_{2}(\bmod \mathfrak{p})$, so good reduction implies $f^{n-m}\left(\beta_{1}\right) \equiv \beta_{2}(\bmod \mathfrak{p})$, equivalently $v_{\mathfrak{p}}\left(f^{n-m}\left(\beta_{1}\right)-\beta_{2}\right)>0$. Applying Lemma 5.1, we have

$$
\begin{aligned}
\sum_{\mathfrak{p} \in \mathcal{X}(n)} N_{\mathfrak{p}} & \leq \sum_{m=1}^{n-B_{\delta}} \sum_{v_{\mathfrak{p}}\left(f^{m}(\gamma)-\beta_{1}\right)>0} N_{\mathfrak{p}}+\sum_{m=n-B_{\delta}+1}^{n-1} \sum_{v_{\mathfrak{p}}\left(f^{n-m}\left(\beta_{1}\right)-\beta_{2}\right)>0} N_{\mathfrak{p}}+O_{\delta}(1) \\
& \leq \delta d^{n} h_{f}(\gamma)+\sum_{i=1}^{B_{\delta}-1} \sum_{v_{\mathfrak{p}}\left(f^{i}\left(\beta_{1}\right)-\beta_{2}\right)>0} N_{\mathfrak{p}}+O_{\delta}(1) .
\end{aligned}
$$

Because $f^{i}\left(\beta_{1}\right)-\beta_{2} \neq 0$ for all $i$, the remaining sum of $N_{\mathfrak{p}}$ comprises a finite set of primes $\mathfrak{p}$ depending on $B_{\delta}$ (and thus on $\delta$ ), so it can be absorbed into the $O_{\delta}(1)$ term.

Lemma 5.3. Let $f \in K[x]$ with $d=\operatorname{deg}(f) \geq 2$. Let $\gamma_{1}, \gamma_{2} \in K$ with $h_{f}\left(\gamma_{2}\right)>0$, and suppose there are integers $\ell_{1}>\ell_{2}$ such that $f^{\ell_{1}}\left(\gamma_{1}\right)=$ $f^{\ell_{2}}\left(\gamma_{2}\right)$. Let $\beta_{1}, \beta_{2} \in K$ with $\beta_{2} \notin \mathcal{O}_{f}\left(\beta_{1}\right)$. For $n>0$, let $\mathcal{Y}(n)$ denote the set of primes $\mathfrak{p}$ such that

$$
\min \left(v_{\mathfrak{p}}\left(f^{m}\left(\gamma_{1}\right)-\beta_{1}\right), v_{\mathfrak{p}}\left(f^{n}\left(\gamma_{2}\right)-\beta_{2}\right)\right)>0
$$

for some $0<m \leq n$. Then for any $\delta>0$, we have

$$
\sum_{\mathfrak{p} \in \mathcal{Y}(n)} N_{\mathfrak{p}} \leq \delta d^{n} h_{f}\left(\gamma_{2}\right)+O_{\delta}(1)
$$

for all $n$.

Proof. The finitely many primes that contribute to the sum for $n<\ell_{1}$ or $m<\ell_{1}$ can be absorbed into the $O_{\delta}(1)$ term, as can the primes $\mathfrak{p}$ for which $f$ has bad reduction at $\mathfrak{p}$. So assume that $f$ has good reduction at $\mathfrak{p}, n \geq \ell_{1}$, $m \geq \ell_{1}, f^{m}\left(\gamma_{1}\right) \equiv \beta_{1}(\bmod \mathfrak{p})$, and $f^{m}\left(\gamma_{1}\right) \equiv \beta_{2}(\bmod \mathfrak{p})$ for some $m \leq n$. Let $m^{\prime}=m-\ell_{1}+\ell_{2}$. Then

$$
f^{m^{\prime}}\left(\gamma_{2}\right)=f^{m-\ell_{1}}\left(f^{\ell_{2}}\left(\gamma_{2}\right)\right)=f^{m-\ell_{1}}\left(f^{\ell_{1}}\left(\gamma_{1}\right)\right)=f^{m}\left(\gamma_{1}\right) \equiv \beta_{1} \quad(\bmod \mathfrak{p}) .
$$

So $\min \left(v_{\mathfrak{p}}\left(f^{m^{\prime}}\left(\gamma_{2}\right)-\beta_{1}\right), v_{\mathfrak{p}}\left(f^{n}\left(\gamma_{2}\right)-\beta_{2}\right)\right)>0$, and also $0<m^{\prime}<n$ because $\ell_{2}-\ell_{1}<0$. We are done by applying Lemma 5.2 to bound the contribution to the sum from such $\mathfrak{p}$.

Lemma 5.4 will be used to treat the case of odd polynomials, which require special attention.

Lemma 5.4. Let $f \in K[x]$ with $d=\operatorname{deg}(f) \geq 3$. Suppose that $f$ is odd (i.e. that $f(-x)=-f(x))$. Let $\gamma \in K \backslash\{0\}$ with $h_{f}(\gamma)>0$. Let $\beta_{1}, \beta_{2} \in K \backslash\{0\}$ be such that $-\beta_{1} \neq \beta_{2}$ and $\beta_{2} \notin \mathcal{O}_{f}\left(-\beta_{1}\right)$. For $n>0$, let $\mathcal{Z}(n)$ denote the set of primes $\mathfrak{p}$ such that

$$
\min \left(v_{\mathfrak{p}}\left(f^{m}(-\gamma)-\beta_{1}\right), v_{\mathfrak{p}}\left(f^{n}(\gamma)-\beta_{2}\right)\right)>0
$$


for some $0<m \leq n$. Then for any $\delta>0$, we have

$$
\sum_{\mathfrak{p} \in \mathcal{Z}(n)} N_{\mathfrak{p}} \leq \delta d^{n} h_{f}(\gamma)+O_{\delta}(1)
$$

for all $n$.

Proof. Since $f$ is odd, we have $f^{m}(-\gamma)=-f^{m}(\gamma)$ for all $m$. Thus, if

$$
\min \left(v_{\mathfrak{p}}\left(f^{n}(-\gamma)-\beta_{1}\right), v_{\mathfrak{p}}\left(f^{n}(\gamma)-\beta_{2}\right)\right)>0
$$

then $v_{\mathfrak{p}}\left(\beta_{1}+\beta_{2}\right)>0$, which restricts such $\mathfrak{p}$ to a finite set as $\beta_{1}+\beta_{2} \neq 0$. Since $v_{\mathfrak{p}}\left(f^{m}(-\gamma)-\beta_{1}\right)=v_{\mathfrak{p}}\left(f^{m}(\gamma)-\left(-\beta_{1}\right)\right)$ for any prime $\mathfrak{p}$, applying Lemma 5.2 with $-\beta_{1}$ in place of $\beta_{1}$ finishes the proof.

Lemma 5.5 is the "Roth- $a b c$ " estimate mentioned in the introduction. For a number field $K$, it is conditional on the $a b c$ conjecture for $K$. As we do not make further use of the $a b c$ conjecture, we refer the reader to [BT18] for its precise statement.

Lemma 5.5. Let $f \in K[x]$ with $d=\operatorname{deg}(f) \geq 3$. Let $\gamma, \beta \in K$ be such that $\beta \notin \mathcal{O}_{f}(\gamma)$. If $K$ is a number field, assume the abc-conjecture for $K$. If $K$ is a function field, assume that $f$ is not isotrivial. Then for every $\epsilon>0$, there exists a constant $C_{\epsilon}$ such that

$$
\sum_{v_{\mathfrak{p}}\left(f^{n}(\gamma)-\beta\right)=1} N_{\mathfrak{p}} \geq(d-\epsilon) d^{n-1} h_{f}(\gamma)+C_{\epsilon} .
$$

Proof. See Propositions 3.4 and 4.2 in [GNT13.

\section{Ramification And Galois theory}

Let $f(x)=x^{3}-3 a^{2} x+b$ with $a, b \in K$. In this section we define Condition $R$ and Condition $U$ in terms of primes dividing certain elements of $K$ related to the forward orbits of $a$ and $-a$. In Proposition 6.4 and 6.5 we show that these conditions control ramification in the extensions $K(\beta) \subseteq K_{n}(\beta)$, with consequences for the Galois theory of these extensions. We begin with the following standard lemma from Galois theory.

Lemma 6.1. Let $L_{1}, \ldots, L_{n}$ and $M$ be fields all contained in some larger field. Assume that $L_{1}, \ldots, L_{n}$ are finite extensions of $M$.

(i) If $L_{1}, L_{2}$ are Galois over $M$ with $L_{1} \cap L_{2}=M$, then $L_{1} L_{2}$ is Galois over $L_{2}$ and $\operatorname{Gal}\left(L_{1} L_{2} / L_{2}\right) \cong \operatorname{Gal}\left(L_{1} / M\right)$.

(ii) If $L_{1}, \ldots, L_{n}$ are Galois over $M$ with $L_{i} \cap \prod_{j \neq i} L_{j}=M$ for each $i$, then $\operatorname{Gal}\left(\prod_{i=1}^{n} L_{i} / M\right) \cong \prod_{i=1}^{n} \operatorname{Gal}\left(L_{i} / M\right)$.

Definition 6.2. Let $\beta \in \bar{K}$. We say that a prime $\mathfrak{p}$ of $K(\beta)$ satisfies Condition $\mathbf{R}$ at $\beta$ for $n$ if the following hold:

(a) $f$ has good separable reduction at $\mathfrak{p}$;

(b) $v_{\mathfrak{p}}\left(f^{i}(-a)-\beta\right)=0$ for all $0 \leq i \leq n$;

(c) $v_{\mathfrak{p}}\left(f^{i}(a)-\beta\right)=0$ for all $0 \leq i<n$; 
(d) $v_{\mathfrak{p}}\left(f^{n}(a)-\beta\right)=1$;

(e) $v_{\mathfrak{p}}(\beta)=0$.

Definition 6.3. Let $\beta \in \bar{K}$. We say that a prime $\mathfrak{p}$ of $K(\beta)$ satisfies Condition $\mathbf{U}$ at $\beta$ for $n$ if the following hold:

(a) $f$ has good separable reduction at $\mathfrak{p}$;

(b) $v_{\mathfrak{p}}\left(f^{i}(a)-\beta\right)=v_{\mathfrak{p}}\left(f^{i}(-a)-\beta\right)=0$ for all $0 \leq i \leq n$;

(c) $v_{\mathfrak{p}}(\beta)=0$.

Proposition 6.4. Let $\beta \in \bar{K}$. Let $\mathfrak{p}$ be a prime of $K(\beta)$ that satisfies condition $U$ at $\beta$ for $n$. Then $\mathfrak{p}$ is unramified in $K_{n}(\beta)$.

Proof. This follows immediately from [BT18, Proposition 3.1]. The proof in [BT18] is stated for $\beta \in K$, but nothing changes if we allow $\beta \in \bar{K}$ and replace $K$ with $K(\beta)$.

Proposition 6.5. Let $\beta \in \bar{K}$. Suppose that $\mathfrak{p}$ is a prime of $K(\beta)$ that satisfies Condition $R$ at $\beta$ for $n$ and that $f^{n}(x)-\beta$ is irreducible over $K(\beta)$. Then

$$
\operatorname{Gal}\left(K_{n}(\beta) / K_{n-1}(\beta)\right) \cong\left(S_{3}\right)^{3^{n-1}}
$$

the direct product of $3^{n-1}$ copies of $S_{3}$. Furthermore, $\mathfrak{p}$ does not ramify in $K_{n-1}(\beta)$ and any field $E$ such that $K_{n-1}(\beta) \subsetneq E \subset K_{n}(\beta)$ must ramify over $\mathfrak{p}$.

Proof. Since Condition $\mathrm{R}$ at $\beta$ for $n$ implies Condition $\mathrm{U}$ at $\beta$ for $n-1$, Proposition 6.4 implies that $\mathfrak{p}$ does not ramify in $K_{n-1}(\beta)$. Now, consider the map $\bar{f}$ on $\mathbb{P}^{1}\left(k_{\mathfrak{p}}\right)$ that comes from reducing $f$ at $\mathfrak{p}$. For any $z \in K$ with $v_{\mathfrak{p}}(z) \geq 0$, we let $\bar{z}$ denote its reduction at $\mathfrak{p}$. The critical points of $\bar{f}$ are then $\bar{a}$ and $-\bar{a}$. Then, by (c) and (d) of Condition R, we see that every point in $\bar{f}^{-(n-1)}(\bar{\beta})$ has ramification index one over $\bar{\beta}$ with respect to $\bar{f}^{n-1}$. Since $\bar{f}(\bar{a}) \in \bar{f}^{-(n-1)}(\bar{\beta})$ and $\bar{f}(-\bar{a}) \notin \bar{f}^{-(n-1)}(\bar{\beta})$ by (b) of Condition R, we see then that $\bar{a}$ has multiplicity 2 as a root of $\bar{f}^{n}(x)-\bar{\beta}$ and that $\bar{f}^{n}(x)-\bar{\beta}$ has no multiple roots besides $\bar{a}$. Thus, we see the reduction of $f^{n}(x)-\beta$ at $\mathfrak{p}$ can be written as

$$
\bar{f}^{n}(x)-\bar{\beta}=(x-\bar{a})^{2} h(x)
$$

where $h \in k_{\mathfrak{p}}[x]$ is coprime to $(x-\bar{a})$ and has no repeated roots.

Let $z_{1}, \ldots, z_{3^{n-1}}$ be the roots of $f^{n-1}(x)-\beta$. Then $f^{n}(x)-\beta$ factors as

$$
f^{n}(x)-\beta=\prod_{i=1}^{3^{n-1}} f(x)-z_{i}
$$

in $K_{n-1}(\beta)$. For each $j$, let $L_{j}$ denote the splitting field of $f(x)-z_{j}$ over $K\left(z_{j}\right)$, and let $M_{j}$ denote the splitting field of $f(x)-z_{j}$ over $K_{n-1}(\beta)$. We see that $M_{j}=K_{n-1} \cdot L_{j}$. By Capelli's Lemma, each $f(x)-z_{j}$ is irreducible over $K\left(z_{j}\right)$.

Note that the $z_{i}$ are distinct modulo $\mathfrak{p}$ since $\bar{f}^{n-1}$ does not ramify at any $\bar{z}_{i}$, as noted above. Let $\mathfrak{q}$ be a prime of $K_{n-1}(\beta)$ that lies over $\mathfrak{p}$, and let $\mathfrak{m}$ be 
a prime of $M_{i}$ lying over q. By (6.5.2) and (6.5.1), there is exactly one $i$ such that $f(x)-z_{i}$ has multiple roots modulo $\mathfrak{q}$, and at this $i$, the polynomial $f(x)-z_{i}$ has a root of multiplicity 2 modulo $\mathfrak{q}$. Then, for $k \neq i$, we see that $\mathfrak{q}$ does not ramify in $M_{k}$. As for its ramification in $M_{i}$, we have $f(x)-z_{i} \equiv$ $\left(x-w_{1}\right)^{2}\left(x-w_{2}\right)(\bmod \mathfrak{m})$ where $w_{1} \equiv a(\bmod \mathfrak{m})$ and $w_{2} \not \equiv a(\bmod \mathfrak{m})$. Now, $v_{\mathfrak{q}}\left(f(a)-z_{i}\right)=1$ since $v_{\mathfrak{p}}\left(f^{n}(a)-\beta\right)=1$ by $(\mathrm{d})$ of Condition $\mathrm{R}$ and $\mathfrak{q}$ does not ramify over $\mathfrak{p}$. On the other hand, $v_{\mathfrak{m}}\left(f(a)-z_{i}\right)=2 v_{\mathfrak{m}}\left(a-w_{1}\right)$, so we see that $\mathfrak{m}$ ramifies over $\mathfrak{p}$ with ramification index 2 . As $\mathfrak{m}$ is unramified in $K\left(z_{i}\right)$, we have that $\mathfrak{m} \cap K\left(z_{i}\right)$ also ramifies over $\mathfrak{p}$ with ramification index 2. Since $f(x)-z_{i}$ is irreducible over $K\left(z_{i}\right)$, this implies that $\left[L_{i}: K\left(z_{i}\right)\right]=6$, i.e. $\operatorname{Gal}\left(L_{i} / K\left(z_{i}\right)\right) \cong S_{3}$. Then $\operatorname{Gal}\left(M_{i} / K_{n-1}(\beta)\right)$ is a normal subgroup of $S_{3}$ (recall $M_{i}=L_{i} \cdot K_{n-1}(\beta)$ ) that contains the order 2 inertia subgroup $I(\mathfrak{m} / \sigma \mathfrak{q})$, and so $\operatorname{Gal}\left(M_{i} / K_{n-1}(\beta)\right) \cong S_{3}$ as well. Thus, $\operatorname{Gal}\left(M_{i} / K_{n-1}(\beta)\right)$ is generated by the three conjugates of $I(\mathfrak{m} / \sigma \mathfrak{q})$, and any field $E^{\prime}$ such that $K_{n-1}(\beta) \subsetneq E^{\prime} \subset M_{i}$ must ramify over $\mathfrak{q}$.

Since $f^{n}(x)-\beta$ is irreducible over $K(\beta)$, it follows easily that $f^{n-1}(x)-\beta$ is irreducible over $K(\beta)$. So all of the $z_{k}$ for which $f^{n-1}\left(z_{k}\right)=\beta$ are conjugate to each other, i.e. for each $1 \leq i, j \leq 3^{n-1}$, there exists $\sigma \in G_{n-1}(\beta)$ such that $\sigma z_{i}=z_{j}$. Then $\sigma \mathfrak{q}$ ramifies in $M_{j}$ with ramification index 2 and does not ramify in $M_{k}$ for $k \neq j$. As above, $\operatorname{Gal}\left(M_{j} / K_{n-1}(\beta)\right)$ is isomorphic to $S_{3}$ and is generated by inertia groups of the form $I(\mathfrak{m} / \sigma \mathfrak{q})$ for primes $\mathfrak{m}$ of $M_{j}$ lying over $\sigma \mathfrak{q}$. Hence any field $E^{\prime}$ such that $K_{n-1}(\beta) \subsetneq E^{\prime} \subset M_{j}$ must ramify over $\sigma \mathfrak{q}$. Since $\sigma \mathfrak{q}$ does not ramify in $M_{k}$ for $k \neq j$, we must therefore have $M_{j} \cap \prod_{k \neq j} M_{k}=K_{n-1}(\beta)$. Thus by Lemma 6.1 the Galois $\operatorname{group} \operatorname{Gal}\left(K_{n}(\beta) / K_{n-1}(\beta)\right)$ is isomorphic to $\left(S_{3}\right)^{3^{n-1}}$.

Each inertia group $I(\mathfrak{m} / \sigma \mathfrak{q})$ for $\mathfrak{m}$ a prime of $M_{j}$ extends to an inertia group $I\left(\mathfrak{m}^{\prime} / \sigma \mathfrak{q}\right)$ for a prime $\mathfrak{m}^{\prime}$ of $K_{n}(\beta)$ lying over $\mathfrak{m}$; the inertia group $I\left(\mathfrak{m}^{\prime} / \sigma \mathfrak{q}\right)$ restricts to $I(\mathfrak{m} / \sigma \mathfrak{q})$ on $M_{j}$ and to the identity on $M_{k}$ for $k \neq$ $j$. Thus, $\operatorname{Gal}\left(K_{n}(\beta) / K_{n-1}(\beta)\right)$ is generated by inertia groups of the form $I\left(\mathfrak{m} / \mathfrak{q}^{\prime}\right)$ for primes $\mathfrak{m}^{\prime}$ of $K_{n}(\beta)$ and primes $\mathfrak{q}^{\prime}$ of $K_{n-1}(\beta)$ lying over $\mathfrak{p}$. Hence, any field $E$ such that $K_{n-1}(\beta) \subsetneq E \subset K_{n}(\beta)$ must ramify over a prime of $K_{n-1}(\beta)$ lying over $\mathfrak{p}$ and thus must ramify over $\mathfrak{p}$, as desired.

Before stating the last result from Galois theory we need, we need a little notation.

Definition 6.6. Let $K$ be a field, let $f \in K[x]$, and let $\boldsymbol{\alpha}=\left(\alpha_{1}, \ldots, \alpha_{s}\right)$, where $\alpha_{i} \in \bar{K}$. We define $K_{n}(\boldsymbol{\alpha})$ to be $\prod_{i=1}^{s} K\left(f^{-n}\left(\alpha_{i}\right)\right)$.

With this notation we have the following.

Proposition 6.7. Let $\boldsymbol{\alpha}=\left(\alpha_{1}, \ldots, \alpha_{s}\right) \subseteq L$ for $L$ a finite extension of $K$. Suppose there exist primes $\mathfrak{p}_{1}, \ldots, \mathfrak{p}_{s}$ of $L$ such that

(a) $\mathfrak{p}_{i} \cap K\left(\alpha_{i}\right)$ satisfies Condition $R$ at $\alpha_{i}$ for $n$;

(b) $\mathfrak{p}_{i} \cap K\left(\alpha_{j}\right)$ satisfies Condition $U$ at $\alpha_{j}$ for $n$ for all $j \neq i$;

(c) $\mathfrak{p}_{i} \cap K\left(\alpha_{i}\right)$ does not ramify in $L$; and

(d) $f^{n}(x)-\alpha_{i}$ is irreducible over $K\left(\alpha_{i}\right)$ for $i=1, \ldots s$. 
Then $\operatorname{Gal}\left(K_{n}(\boldsymbol{\alpha}) / K_{n-1}(\boldsymbol{\alpha})\right) \cong S_{3}^{s 3^{n-1}}$.

Proof. By Proposition 6.5, for $i=1, \ldots, s$, we have

$$
\operatorname{Gal}\left(K_{n}\left(\alpha_{i}\right) / K_{n-1}\left(\alpha_{i}\right)\right) \cong S_{3}^{3^{n-1}} .
$$

Also by Proposition 6.5. for each $i$, the prime $\mathfrak{p}_{i}$ does not ramify in $K_{n-1}\left(\alpha_{i}\right)$, but any field $E$ with $K_{n-1}\left(\alpha_{i}\right) \subsetneq E \subset K_{n}\left(\alpha_{i}\right)$ must ramify over $\mathfrak{p}_{i}$. Let $L_{i}$ denote $K_{n}\left(\alpha_{i}\right) \cdot K_{n-1}(\boldsymbol{\alpha})$. By Proposition 6.4 and condition (c), the prime $\mathfrak{p}_{i}$ does not ramify in $K_{n-1}(\boldsymbol{\alpha})$ or in $L_{j}$ for $j \neq i$, so we must have $K_{n}\left(\alpha_{i}\right) \cap \prod_{j \neq i} L_{j}=K_{n-1}\left(\alpha_{i}\right)$ and $K_{n}\left(\alpha_{i}\right) \cap K_{n-1}(\boldsymbol{\alpha})=K_{n-1}(\alpha)$. Thus,

$\operatorname{Gal}\left(L_{i} \cdot \prod_{j \neq i} L_{j} / \prod_{j \neq i} L_{j}\right) \cong \operatorname{Gal}\left(L_{i} / K_{n-1}(\boldsymbol{\alpha})\right) \cong \operatorname{Gal}\left(K_{n}\left(\alpha_{i}\right) / K_{n-1}\left(\alpha_{i}\right)\right) \cong S_{3}^{3^{n-1}}$

by Lemma 6.1(i), since $L_{i} \cdot \prod_{j \neq i} L_{j}=K_{n}\left(\alpha_{i}\right) \cdot \prod_{j \neq i} L_{j}$. This means that

$$
6^{3^{n}-1}=\left[L_{i}: K_{n-1}(\boldsymbol{\alpha})\right] \geq\left[L_{i}: L_{i} \cap \prod_{j \neq i} L_{j}\right] \geq\left[L_{i} \cdot \prod_{j \neq i} L_{j}: \prod_{j \neq i} L_{j}\right]=6^{3^{n}-1} .
$$

Thus, we have $L_{i} \cap \prod_{j \neq i} L_{j}=K_{n-1}(\boldsymbol{\alpha})$. Using the fact that $L_{i} \cdot \prod_{j \neq i} L_{j}=$ $K_{n}(\boldsymbol{\alpha})$ and applying Lemma 6.1(ii) then finishes our proof.

\section{The CASE OF NUMBER FIELDS}

Throughout this section, $K$ will denote a number field. The main result of the section is Lemma 7.6, which handles the height estimates needed for the proof of Theorem 1.1. We will use the following form of Vojta's conjecture for $K$ Voj87, Conjecture 3.4.3].

Conjecture 7.1 (Vojta). Let $V$ be a smooth projective variety over a number field. Let $\mathcal{K}$ be the canonical divisor of $V$ and let $A$ be an ample normal crossings divisor, with associated height functions $h_{\mathcal{K}}$ and $h_{A}$. For any $\epsilon>0$, there is a proper Zariski-closed $X_{\epsilon} \subset V$ and a constant $C_{\epsilon}=C_{\epsilon}(V, K, A)$ such that

$$
h_{\mathcal{K}}(x) \leq h_{A}(x)+C_{\epsilon}
$$

for all $x \in V(K) \backslash X_{\epsilon}(K)$.

A sequence $\left(a_{n}\right)$ of points on a variety $V$ is said to be generic if for every proper Zariski-closed subset $X \subset V$, there exists $N \in \mathbb{N}$ such that $a_{n} \notin X$ for all $n>N$. Note that this is a stronger condition than the set of all points in the sequence being Zariski-dense in $V$. A key step in our proof for number fields uses Theorem 7.2 , a conditional bound on "dynamical gcds" for generic orbits due to Huang [Hua17, Theorem 2.4]. For any valuation $v \in M_{K}$, let $v^{+}(a)=\max (v(a), 0)$. For $a, b \in K$, define

$$
h_{g c d}^{0}(a, b)=\sum_{\mathfrak{p} \text { of } \mathfrak{o}_{K}} N_{\mathfrak{p}} \min \left(v_{\mathfrak{p}}^{+}(a), v_{\mathfrak{p}}^{+}(b)\right)
$$


and

$$
h_{g c d}(a, b)=h_{g c d}^{0}(a, b)+\frac{1}{[K: \mathbb{Q}]} \sum_{v \in M_{K}^{\infty}} \min \left(v^{+}(a), v^{+}(b)\right),
$$

where $N_{\mathfrak{p}}$ is as in Section 5 and $M_{K}^{\infty}$ is the set of archimedean places of $K$. Observe that $h_{g c d}^{0}(a, b)$ is a finite sum where the only positive contributions come from primes $\mathfrak{p}$ such that both $v_{\mathfrak{p}}(a)>0$ and $v_{\mathfrak{p}}(b)>0$. Clearly $h_{g c d}^{0}(a, b) \leq h_{g c d}(a, b)$.

The point $z \in \mathbb{P}^{1}(\bar{K})$ is said to be exceptional for $f \in K(x)$ if the backward orbit of $z$ under $f$ is finite, i.e. if there are only finitely many $y \in \mathbb{P}^{1}(\bar{K})$ such that $f^{n}(y)=z$ for some $n \geq 1$. It is easy to show that, up to conjugation by Möbius transformations, $z$ is exceptional for $f$ if and only if either $f$ is a polynomial and $z=\infty$ or $f(x)=x^{d}$ for some nonzero $d \in \mathbb{Z}$ and $z \in\{0, \infty\}$. With this notation, we can state Huang's result, which may be seen as a dynamical analog of work of Bugeaud, Corvaja, and Zannier BCZ03, CZ05. We note that Huang's proof follows ideas of Silverman Sil05, which connect the result of Bugeaud, Corvaja, and Zannier with a special case of Vojta's conjecture.

Theorem 7.2. Assume Vojta's conjecture for blowups of $\mathbb{P}^{1} \times \mathbb{P}^{1}$. Let $f, g \in$ $K(x)$ with $\operatorname{deg} f=\operatorname{deg} g$ and let $a, b, c, d \in K$ such that $c$ is not exceptional for $f$ and $d$ is not exceptional for $g$. Suppose that the sequence $\left(f^{n}(a), g^{n}(b)\right)$ is generic in $\mathbb{A}^{2}(K)$. Then for every $\epsilon>0$, there exists a constant $C_{\epsilon}=$ $C_{\epsilon}(a, b, c, d, f, g)$ such that

$$
h_{g c d}\left(f^{n}(a)-c, g^{n}(b)-d\right)<\epsilon \max \left(h_{f}(a), h_{g}(b)\right)(\operatorname{deg} f)^{n}+C_{\epsilon}
$$

for all $n \geq 1$.

If the sequence $\left(f^{n}(a), f^{n}(-a)\right)$ is generic in $\mathbb{A}^{2}(K)$, then we will apply Theorem 7.2 to demonstrate the existence of primes that satisfy Condition $\mathrm{R}$ of Section 6.1. We also need to handle the possibility that this sequence is not generic. This is done using the following result of Xie [Xie15], which proves the Dynamical Mordell-Lang Conjecture (see e.g. [GTZ08, BGKT12]) for polynomial endomorphisms of $\mathbb{A}^{2}(\overline{\mathbb{Q}})$. This will be used to prove Lemma 7.5 . which shows that in the non-generic case, the sequence must lie on a curve of a very restricted form.

Theorem 7.3. Let $F: \mathbb{A}^{2} \rightarrow \mathbb{A}^{2}$ be a polynomial endomorphism defined over $\overline{\mathbb{Q}}$. Let $C$ be an irreducible curve in $\mathbb{A}^{2}$ and let $\alpha$ be a closed point in $\mathbb{A}^{2}(\overline{\mathbb{Q}})$. Then the set $\left\{n \in \mathbb{N}: F^{n}(\alpha) \in C\right\}$ is a finite union of arithmetic progressions.

Let $f \in K[x]$ be a polynomial of degree $d \geq 2$. We say $f$ is in normal form if $f$ is monic and the second highest degree term of $f$ has a coefficient of zero. That is, a normal form $f$ can be written

$$
f(x)=x^{d}+a_{d-2} x^{d-2}+a_{d-3} x^{d-3}+\cdots+a_{1} x+a_{0} .
$$


It is easy to show for any nonlinear $f \in K[x]$, there exists a linear $\sigma \in \bar{K}[x]$ such that $\sigma \circ f \circ \sigma^{-1}(x)$ is in normal form.

We will use Lemma 7.4 to determine the linear polynomials that commute with iterates of normal form polynomials. We say $f$ is of $g a p k$ if the second highest degree term in $f$ is of degree $\operatorname{deg} f-k$.

Lemma 7.4. Suppose that $f \in K[x]$ of degree $d \geq 2$ is in normal form of gap $k$. Then $f^{n}$ is in normal form of gap $k$ for all $n \geq 1$.

Proof. Write

$$
f(x)=x^{d}+a_{d-k} x^{d-k}+\text { terms of degree less than } d-k .
$$

By induction we have

$$
f^{n}(x)=x^{d^{n}}+d^{n-1} a_{d-k} x^{d^{n}-k}+\text { terms of degree less than } d^{n}-k .
$$

Lemma 7.5. Let $f(x)=x^{3}-3 a^{2} x+b \in K[x]$ with $a \neq 0$ and $b \neq 0$. Suppose that $f$ is not PCF. If the sequence $\left(f^{n}(a), f^{n}(-a)\right)$ is not generic in $\mathbb{A}^{2}(K)$, then there exist $m, n \in \mathbb{N}$ such that $f^{m}(a)=f^{n}(-a)$.

Proof. Let $F: \mathbb{A}^{2} \longrightarrow \mathbb{A}^{2}$ be given by $F(x, y)=(f(x), f(y))$. If the sequence $F^{n}(a,-a)$ is not generic in $\mathbb{A}^{2}$, there is a curve $C \subseteq \mathbb{A}^{2}$ such that $F^{n}(a,-a) \in$ $C$ for infinitely many $n$. By Xie's proof [Xie15] of the dynamical MordellLang conjecture for $\mathbb{A}^{2}(K)$, it follows that there are integers $m_{0} \geq 0$ and $m_{1}>0$ such that $F^{m_{0}+k m_{1}}(a,-a) \in C$ for all integers $k \geq 0$. Therefore $\left\{F^{m_{0}+m_{1} k}(a,-a): k \geq 0\right\} \subseteq C$, and the Zariski closure of this set is $F^{m_{1}}$ invariant. As $f$ is not $\mathrm{PCF}$, this set is infinite, so $C$ is $F^{m_{1}}$-invariant.

By the chain rule, critical points of $f$ are also critical points of $f^{m_{1}}$. Thus, as we assumed $f$ is not PCF, it follows that $f^{m_{1}}$ is not PCF either. In particular, $f^{m_{1}}$ is not a power map, Chebyshev polynomial, or negative Chebyshev polynomial. Therefore by work of Medvedev and Scanlon [MS14, the $F^{m_{1}}$-invariant curve $C$ is the graph of a polynomial that commutes with an iterate of $f$. That is, $C$ is given by an equation of the form $y=p(x)$ or $x=p(y)$ where $p \in K[x]$ commutes with with some iterate of $f$, that is, $f^{t} \circ p=p \circ f^{t}$ for some $t>0$. Moreover, Ritt's theorem on commuting polynomials [Rit23] implies that $p(x)=L \circ h^{r}(x)$ for some $r>0$ and $L, h \in$ $K[x]$ where $h^{k}=f^{\ell}$ for some $k$ and $\ell$, and $L$ is a linear polynomial that commutes with some iterate of $f$. By Lemma 7.4, as $f$ is in normal form with gap $2, f^{n}$ is also in normal form with gap 2 . So the only nonidentity linear polynomial $L$ that can commute with any iterate of $f^{n}$ is $L(x)=-x$. Now, by [Rez79], if $f^{n}$ is odd, then $f$ is odd, but we have assumed that $b \neq 0$, so $f$ is not odd. Thus, any polynomial that commutes with $f$ shares a common iterate with $f$.

Ritt's classification of complex polynomials with a common iterate Rit20] gives

$$
f(x)=-B+\epsilon_{1} g^{n_{1}}(x+B)
$$


and

$$
h(x)=-B+\epsilon_{2} g^{n_{2}}(x+B)
$$

where $B \in \mathbb{C}, g(x) \in x^{r} \mathbb{C}\left[x^{s}\right]$ for some $s \geq 1$, and $\epsilon_{1}^{s}=\epsilon_{2}^{s}=1$. Note that $s$ can be taken to be the largest integer such that the exponents occurring in $g$ form an arithmetic progression of modulus $s$. We must have $n_{1}=1$, since $f$ has prime degree. Furthermore, we must have $s=1$, since if $s \geq 2$, then $B=$ 0 (since $f$ is in normal form already), which would mean that $g$ is a multiple of $f$, and the linear term of $f$ would be zero, contradicting our assumption that $a \neq 0$. It follows then that $\epsilon_{1}=\epsilon_{2}=1$. Now, let $\sigma(x)=x+B$. Then $f=\sigma^{-1} g \sigma$ and $h=\sigma^{-1} g^{n_{2}} \sigma$, so $h=f^{n_{2}}$. Therefore, $C$ is given by an equation of the form $y=f^{r}(x)$ or $x=f^{r}(y)$. Since $\left(f^{m_{0}}(a), f^{m_{0}}(-a)\right) \in C$, we see then that there are $m, n$ such that $f^{m}(a)=f^{n}(-a)$, as desired.

Lemma 7.6. Let $f(x)=x^{3}-3 a^{2} x+b \in K[x]$ with $a \neq 0$ and $b \neq 0$. Let $\beta_{1}, \beta_{2} \in K$. Assume both the abc-conjecture for $K$ and Vojta's conjecture for blowups of $\mathbb{P}^{1} \times \mathbb{P}^{1}$. Suppose that $f$ is not PCF and that $h_{f}(a) \geq h_{f}(-a)$. Suppose that $f^{i}(a) \neq \beta_{1}$ and $f^{i}(-a) \neq \beta_{2}$ for any $i>0$. Let $\mathcal{Y}(n)$ be the set of primes $\mathfrak{p}$ of $K$ such that

$$
\min \left(v_{\mathfrak{p}}\left(f^{n}(a)-\beta_{1}\right), v_{\mathfrak{p}}\left(f^{m}(-a)-\beta_{2}\right)\right)>0
$$

for some $1 \leq m \leq n$. Then for any $\delta>0$, we have

$$
\sum_{\mathfrak{p} \in \mathcal{Y}(n)} N_{\mathfrak{p}} \leq \delta 3^{n} h_{f}(a)+O_{\delta}(1)
$$

Proof. First note that $h_{f}(a)>0$, because if $h_{f}(a)=0$ then $h_{f}(-a)=0$ also. But Northcott's Theorem then implies $a$ and $-a$ are both preperiodic for $f$ (see [CS93]). Then we have that $f$ is PCF, which is excluded by assumption.

If $\beta_{1}$ or $\beta_{2}$ were exceptional for $f$, then $f$ would be a power map, again contradicting our assumption that $f$ is not PCF. Thus, if the sequence $\left(f^{n}(a), f^{n}(-a)\right)$ is not generic in $\mathbb{A}^{2}$, then by Theorem 7.5 there are positive integers $\ell_{1}, \ell_{2}$ such that $f^{\ell_{1}}(a)=f^{\ell_{2}}(-a)$, and the conclusion follows from Lemma 5.3. Assume therefore that the sequence $\left(f^{n}(a), f^{n}(-a)\right)$ is generic in $\mathbb{A}^{2}$. Theorem 7.2 and the inequality $h_{g c d}^{0} \leq h_{g c d}$ imply that the conclusion holds in the case where $m=n$, that is,

$$
\sum_{\min \left(v_{\mathfrak{p}}\left(f^{n}(a)-\beta_{1}\right), v_{\mathfrak{p}}\left(f^{n}(-a)-\beta_{2}\right)\right)>0} N_{\mathfrak{p}} \leq \delta 3^{n} h_{f}(a)+O_{\delta}(1) .
$$

For $m<n$, a more delicate argument is required.

By Lemma 5.1, up to possibly replacing $\delta$ by something smaller, there exists $B_{\delta} \leq-\frac{\log \delta}{\log 3}$ such that

$$
\sum_{m=1}^{n-B_{\delta}} \sum_{v_{\mathfrak{p}}\left(f^{m}(-a)-\beta_{2}\right)>0} N_{\mathfrak{p}} \leq \delta 3^{n} h_{f}(-a) \leq \delta 3^{n} h_{f}(a) .
$$

Let $m$ be such that $n-B_{\delta}<m \leq n$. Assume that $f$ has good reduction at $\mathfrak{p}$ (as usual, any contribution from the finitely many primes of bad reduction 
will be absorbed into the $O_{\delta}(1)$ term). Then if $v_{\mathfrak{p}}\left(f^{m}(-a)-\beta_{2}\right)>0$, we have $f^{m}(-a) \equiv \beta_{2}(\bmod \mathfrak{p})$, so

$$
f^{n}(-a)=f^{n-m}\left(f^{m}(-a)\right) \equiv f^{n-m}\left(\beta_{2}\right) \quad(\bmod \mathfrak{p})
$$

where $n-m<B_{\delta}$. For each $j$ with $0 \leq j<B_{\delta}$, let $\mathcal{W}(j)$ be the set of primes $\mathfrak{p}$ of $\mathfrak{o}_{K} \operatorname{such}$ that $\min \left(v_{\mathfrak{p}}\left(f^{n}(a)-\beta_{1}\right), v_{\mathfrak{p}}\left(f^{n}(-a)-f^{j}\left(\beta_{2}\right)\right)>0\right.$. Then

$$
\sum_{\min \left(v_{\mathfrak{p}}\left(f^{n}(a)-\beta_{1}\right), v_{\mathfrak{p}}\left(f^{m}(-a)-\beta_{2}\right)\right)>0} N_{\mathfrak{p}} \leq \sum_{\mathfrak{p} \in \mathcal{W}(n-m)} N_{\mathfrak{p}}
$$

Now we apply Theorem 7.2 to bound the contribution to the sum from the various $\mathcal{W}(j)$ :

$$
\sum_{j=1}^{B_{\delta}-1} \sum_{\mathfrak{p} \in \mathcal{W}(j)} N_{\mathfrak{p}} \leq B_{\delta} \delta h_{f}(a) 3^{n}+C_{\delta} \leq-\frac{\delta \log \delta}{\log 3} h_{f}(a) 3^{n}+C_{\delta},
$$

Adding (7.6.1) and (7.6.2), and observing that $\delta \log \delta \rightarrow 0$ as $\delta \rightarrow 0$, we are done.

\section{The CASE OF FUNCTION FIELDS}

Throughout this section, $K$ will denote a function field of transcendence degree 1 over an algebraic extension of $\mathbb{Q}$. We derive the following from work of Ghioca and Ye [GY16] (see also [FG16]) along with Call-Silverman specialization [CS93].

Proposition 8.1. Suppose that $f(x)=x^{3}-3 a^{2} x+b \in K[x]$ is not isotrivial. Suppose furthermore that $b \neq 0$ and that there are no $i, j>0$ such that $f^{i}(a)=f^{j}(-a)$. Let $c, d \in K$ be such that $f^{\ell}(a) \neq c$ and $f^{\ell}(-a) \neq d$ for all positive integers $\ell$. Then there are at most finitely many places $v$ of $K$ such that there are positive integers $m, n$ with the property that

$$
\min \left(v\left(f^{m}(a)-c\right), v\left(f^{n}(-a)-d\right)\right)>0 .
$$

Recall that a function field $K$ of transcendence degree 1 over an algebraic extension of $\mathbb{Q}$ gives rise to a curve $C$ defined over $\mathbb{Q}$, and that the places of $K$ correspond to closed points in $C(\overline{\mathbb{Q}})$ (when we refer to points of $C(\overline{\mathbb{Q}})$, we will mean closed points with respect to the field of constants of $K$ ). For any element of $c \in K$ and any point $\lambda$ of $C(\overline{\mathbb{Q}})$ such that $c$ does not have a pole at $\lambda$, we let $c_{\lambda}$ denote the specialization of $c$ to $\overline{\mathbb{Q}}$ at $\lambda$ (see CS93] for more details); likewise for a rational function $\varphi \in K(x)$, we let $\varphi_{\lambda}$ denote the specialization of $\varphi$ to $\overline{\mathbb{Q}}(x)$ at $\lambda$ for any $\lambda$ such that the coefficients of $\varphi$ do not have poles at $\lambda$. With notation as above, Ghioca and Ye GY16] proved the following (see also [FG16] for a similar result).

Theorem 8.2. ([GY16]). Let $f(x)=x^{3}-3 a^{2} x+b \in K[x]$ be nonisotrivial. If there is an infinite sequence $\left(\lambda_{i}\right)_{i=1}^{\infty}$ in $C(\overline{\mathbb{Q}})$ such that

$$
\lim _{i \rightarrow \infty} h_{f_{\lambda_{i}}}\left(a_{\lambda_{i}}\right)=\lim _{i \rightarrow \infty} h_{f_{\lambda_{i}}}\left(-a_{\lambda_{i}}\right)=0,
$$

then at least one of the following holds: 
- $b=0$;

- $a$ is preperiodic under $f$;

- $-a$ is preperiodic under $f$; or

- there are $i, j>0$ such that $f^{i}(a)=f^{j}(-a)$.

We now prove a simple lemma that follows from work of Call and Silverman CS93. Note that this lemma works for any rational function of degree greater than one over a function field, not just for cubic polynomials.

Lemma 8.3. Let $\varphi \in K(x)$ have degree $d \geq 2$. Let $y, z \in K$ with $h_{\varphi}(y)>0$. Let $\left(\lambda_{i}\right)_{i=1}^{\infty}$ be a sequence of points of $C(\overline{\mathbb{Q}})$ satisfying $\varphi_{\lambda_{i}}^{n_{i}}\left(y_{\lambda_{i}}\right)=z_{\lambda_{i}}$ for a sequence $\left(n_{i}\right)_{i=1}^{\infty}$ of positive integers with $\lim _{i \rightarrow \infty} n_{i}=\infty$. Then

$$
\lim _{i \rightarrow \infty} h_{\varphi_{\lambda_{i}}}\left(y_{\lambda_{i}}\right)=0 \text {. }
$$

Proof. For each $i$ we have

$$
h_{\varphi_{\lambda_{i}}}\left(y_{\lambda_{i}}\right)=\frac{1}{d^{n_{i}}} h_{\varphi_{\lambda_{i}}}\left(z_{\lambda_{i}}\right)
$$

since $h_{\varphi_{\lambda_{i}}}\left(\varphi_{\lambda_{i}}(x)\right)=d h_{\varphi_{\lambda_{i}}}(x)$ for all $x \in \overline{\mathbb{Q}}$. Let $h_{C}$ be a height function corresponding to a divisor of degree one on $C$. Then, by CS93, Theorem 4.1], we have

$$
\lim _{h_{C}(t) \rightarrow \infty} \frac{h_{\varphi_{t}}\left(y_{t}\right)}{h_{C}(t)}=h_{\varphi}(y)
$$

and

$$
\lim _{h_{C}(t) \rightarrow \infty} \frac{h_{\varphi_{t}}\left(z_{t}\right)}{h_{C}(t)}=h_{\varphi}(z) .
$$

Hence, the $h_{C}\left(\lambda_{i}\right)$ must be bounded for all $i$, since otherwise 8.3.1) would imply that $h_{\varphi}(y)=0$. Now, Theorem 3.1 of CS93. states that

$$
\left|h_{\varphi_{t}}\left(z_{\lambda_{i}}\right)-h_{\varphi}(z)\right| \leq O(1)\left(h_{C}(t)+1\right)
$$

for all $t$ in $C(\overline{\mathbb{Q}})$. As $h_{C}\left(\lambda_{i}\right)$ is bounded, we see that $h_{\varphi_{\lambda_{i}}}\left(z_{\lambda_{i}}\right)$ must be bounded for all $i$. The Lemma then follows immediately from 8.3.1).

Now we can prove Proposition 8.1 .

Proof of Proposition 8.1. If $a$ is preperiodic, then there are only finitely many places $v$ of $K$ such that there is an $m$ for which $v\left(f^{m}(a)-c\right)>0$. Likewise, if $-a$ is preperiodic then there are only finitely many places $v$ such that there is an $n$ for which $v\left(f^{n}(a)-c\right)>0$. Hence, we may assume that neither $a$ nor $-a$ is preperiodic. We now argue by contradiction. Suppose there is an infinite sequence of places $\left(v_{i}\right)_{i=1}^{\infty}$ such that for each $i$, we have $m_{i}, n_{i}$ with the property that

$$
\min \left(v_{i}\left(f^{m_{i}}(a)-c\right), v_{i}\left(f^{n_{i}}(-a)-d\right)\right)>0 .
$$

Let $\lambda_{i}$ be the point in $C(\overline{\mathbb{Q}})$ corresponding to $v_{i}$. Then we have $f_{\lambda_{i}}^{m_{i}}\left(a_{\lambda_{i}}\right)=$ $c_{\lambda_{i}}$ and $f_{\lambda_{i}}^{n_{i}}\left(-a_{\lambda_{i}}\right)=d_{\lambda_{i}}$. Since $c$ is not in the forward orbit of $a$ and $d$ is not in the forward orbit of $-a$, we see that $\lim _{i \rightarrow \infty} m_{i}=\lim _{i \rightarrow \infty} n_{i}=\infty$ 
since any $m_{i}$ or $n_{i}$ can only arise for a finite number of places $v_{i}$. Thus, by Lemma 8.3, we have

$$
\lim _{i \rightarrow \infty} h_{f_{\lambda_{i}}}\left(a_{\lambda_{i}}\right)=\lim _{i \rightarrow \infty} h_{f_{\lambda_{i}}}\left(-a_{\lambda_{i}}\right)=0 .
$$

Theorem 8.2 then gives a contradiction since $b \neq 0$ and there are no $i, j>0$ such that $f^{i}(a)=f^{j}(-a)$.

Remark 8.4. Although Ghioca and Ye only prove Theorem 8.2 over $\overline{\mathbb{Q}}$, their proof works for the algebraic closure of any finitely generated extension of $\mathbb{Q}$. Any finitely generated extension $M$ of $\mathbb{Q}$ can be endowed with a set of places that give rise to appropriate height functions on the algebraic closure of $M$ (see [BG06, Chapter 1], for example), and work of Gubler Gub08 extends Yuan's equidistribution theorem [Yua08] for small points over $\overline{\mathbb{Q}}$ to this context. The authors of both [GY16] and [FG16] have confirmed that their proofs can extended with essentially no modification to prove that Theorem 8.2 holds when $K$ is a function field over any the algebraic closure of any finitely generated extension $M$ of $\mathbb{Q}$ and $h_{f_{\lambda_{i}}}\left( \pm a_{\lambda_{i}}\right)$ are canonical heights associated to a Weil height for $M$. Hence, Proposition 8.1 holds over a function field over any field of constants of characteristic 0 , and the main results of this paper hold in that context as well, since nothing else in our arguments require that the field of constants of $K$ be $\overline{\mathbb{Q}}$.

\section{Proof of Main Theorem}

Let $K$ be a number field or a function field of characteristic 0 of transcendence degree 1 over an algebraic extension of $\mathbb{Q}$. If $K$ is a number field, for the rest of the section we will assume both the $a b c$ conjecture for $K$ and Vojta's conjecture for blowups of $\mathbb{P}^{1} \times \mathbb{P}^{1}$.

The proof of Theorem 1.1 combines the preliminary arguments from throughout the paper with the following proposition, which produces primes with certain ramification behavior in $K_{n}(\beta)$. Recall the definitions of Condition $\mathrm{R}$ and Condition $\mathrm{U}$ from Section 6.1.

Proposition 9.1. Let $f(x)=x^{3}-3 a^{2} x+b \in K[x]$. Assume that $a \neq 0$ and that $f$ is not PCF. Let $L$ be a finite extension of $K$. Let $\alpha_{1}, \ldots, \alpha_{s}$ be distinct elements of $L$ such that $\alpha_{i} \notin \mathcal{O}_{f}(a) \cup \mathcal{O}_{f}(-a)$ and $\alpha_{i} \notin \mathcal{O}_{f}\left(\alpha_{j}\right)$ for all $i, j \in\{1, \ldots, s\}$. If $b=0$, further assume that $\alpha_{i} \notin \mathcal{O}_{f}\left(-\alpha_{j}\right)$ for all $i, j \in\{1, \ldots, s\}$. Then, for all sufficiently large $n$, there exist primes $\mathfrak{p}_{1}, \ldots, \mathfrak{p}_{s}$ of $L$ such that

(a) $\mathfrak{p}_{i} \cap K\left(\alpha_{i}\right)$ satisfies Condition $R$ at $\alpha_{i}$ for $n$;

(b) $\mathfrak{p}_{i} \cap K\left(\alpha_{j}\right)$ satisfies Condition $U$ at $\alpha_{j}$ for $n$ for all $j \neq i$;

(c) $\mathfrak{p}_{i} \cap K\left(\alpha_{i}\right)$ does not ramify in $L$.

Proof. For any $1 \leq i \leq s$, let $\mathcal{A}_{i}(n)$ be the set of primes $\mathfrak{p}$ of $L$ such that (a), (b), and (c) hold. If a prime $\mathfrak{p}$ of $L$ satisfies condition $\mathrm{R}$ or condition $\mathrm{U}$ at $\alpha_{i}$ for $n$, then it is easy to see that the prime $\mathfrak{p} \cap K\left(\alpha_{i}\right)$ of $K\left(\alpha_{i}\right)$ also satisfies condition $\mathrm{R}$ or condition $\mathrm{U}$ at $\alpha_{i}$ for $n$. Therefore we will establish 
Conditions $\mathrm{R}$ and $\mathrm{U}$ for primes of $L$ rather than primes of the various $K\left(\alpha_{i}\right)$, which will make the argument less cumbersome to state. Thus all sums below are indexed by primes of $\mathfrak{o}_{L}$ as in Section 5 .

There are only finitely many primes $\mathfrak{p}$ of $\mathfrak{o}_{L}$ for which $f$ does not have good separable reduction at $\mathfrak{p}, v_{\mathfrak{p}}\left(\alpha_{i}\right) \neq 0$ for some $i$, or $\mathfrak{p} \cap K\left(\alpha_{i}\right)$ ramifies in $L$ for some $\alpha_{i}$. The contributions of these primes to our estimates will be absorbed into the constant term $C_{\delta}^{\prime}$ at the end of the proof.

Choose $i$ and $j$ with $1 \leq i, j \leq s$ (and possibly $i=j$ ). By Lemma 5.5, for any $\delta>0$ there is a constant $C_{\delta}$ such that

$$
\sum_{v_{\mathfrak{p}}\left(f^{n}(a)-\alpha_{i}\right)=1} N_{\mathfrak{p}} \geq(3-\delta) 3^{n-1} h_{f}(a)+C_{\delta} .
$$

Let $\mathcal{X}(n)$ be the set of primes $\mathfrak{p}$ with $\min \left(v_{\mathfrak{p}}\left(f^{n}(a)-\alpha_{j}\right)>0, v_{\mathfrak{p}}\left(f^{m}(a)-\right.\right.$ $\left.\left.\alpha_{i}\right)\right)>0$ for some $1 \leq m \leq n-1$. By Lemma 5.2 with $\gamma=a, \beta_{1}=\alpha_{i}$, and $\beta_{2}=\alpha_{j}$, we have

$$
\sum_{\mathfrak{p} \in \mathcal{X}(n)} N_{\mathfrak{p}} \leq \delta 3^{n} h_{f}(a)+O_{\delta}(1)
$$

Furthermore, if $j \neq i$, then the set of primes $\mathfrak{p}$ such that $\min \left(v_{\mathfrak{p}}\left(f^{n}(a)-\right.\right.$ $\left.\left.\alpha_{i}\right), v_{\mathfrak{p}}\left(f^{n}(a)-\alpha_{j}\right)\right)>0$ is a finite set depending only on $\alpha_{i}$ and $\alpha_{j}$ (and not on $n)$, because $\alpha_{i} \equiv \alpha_{j}(\bmod \mathfrak{p})$ for such $\mathfrak{p}$. So

$$
\sum_{\min \left(v_{\mathfrak{p}}\left(f^{n}(a)-\alpha_{i}\right), v_{\mathfrak{p}}\left(f^{n}(a)-\alpha_{j}\right)\right)>0} N_{\mathfrak{p}}=O_{\delta}(1) .
$$

Let $\mathcal{Y}(n)$ be the set of $\mathfrak{p}$ with $\min \left(v_{\mathfrak{p}}\left(f^{n}(a)-\alpha_{i}\right), v_{\mathfrak{p}}\left(f^{m}(-a)-\alpha_{j}\right)\right)>0$ for some $1 \leq m \leq n$. If $b=0$, then applying Lemma 5.4 with $\beta_{1}=\alpha_{i}$ and $\beta_{2}=\alpha_{j}$ gives

$$
\sum_{\mathfrak{p} \in \mathcal{Y}(n)} N_{\mathfrak{p}} \leq \delta 3^{n} h_{f}(a)+O_{\delta}(1)
$$

If $b \neq 0$ and $K$ is a number field, then applying Lemma 7.6 with $\beta_{1}=\alpha_{i}$ and $\beta_{2}=\alpha_{j}$, again we arrive at 9.1.4. If $b \neq 0$ and $K$ is a function field, then instead of Lemma 7.6, we invoke Proposition 8.1 with $c=\alpha_{i}$ and $d=\alpha_{j}$. This gives us the stronger statement that there are only finitely many primes $\mathfrak{p}$ of $K$ such that both $v_{\mathfrak{p}}\left(f^{n}(a)-\alpha_{i}\right)>0$ and $v_{\mathfrak{p}}\left(f^{m}(-a)-\alpha_{j}\right)>0$ for any $1 \leq m \leq n$. So for function fields, we actually have

$$
\sum_{\mathfrak{p} \in \mathcal{Y}(n)} N_{\mathfrak{p}}=O_{\delta}(1)
$$

though we do not require the full strength of this bound.

Starting with (9.1.1) for some choice of $1 \leq i \leq s$, for each $j$ with $1 \leq j \leq s$ we subtract $(9.1 .2)$ and either $(9.1 .4)$ or $(9.1 .5)$, and for all $j \neq i$ we subtract 9.1.3). This sieves out all $\mathfrak{p} \notin \mathcal{A}_{i}(n)$ from the sum in (9.1.1), and we have

$$
\sum_{\mathfrak{p} \in \mathcal{A}_{i}(n)} N_{\mathfrak{p}} \geq 3^{n} h_{f}(a)(1-\delta / 3-2 s \delta)+C_{\delta}^{\prime}
$$


where $C_{\delta}^{\prime}$ is a constant obtained by combining all the $O_{\delta}(1)$ terms. For sufficiently large $n$, we can choose some $\delta$ to make the RHS positive. Repeating this process for each $i$, we are done.

We are now ready to prove Theorem 1.1. Let $f \in K[x]$ be a degree 3 polynomial and let $\beta \in K$. Assume that $f$ is not postcritically finite, that $\beta$ is not postcritical for $f$, that $(f, \beta)$ is eventually stable, and that $f$ has distinct finite critical points $\gamma_{1}, \gamma_{2}$ with $f^{n}\left(\gamma_{1}\right) \neq f^{n}\left(\gamma_{2}\right)$ for any $n \geq 1$. By Proposition 3.2, we can replace $K$ with a finite extension and replace $f$ with a change of variables so that $f(x)=x^{3}-3 a^{2} x+b$ for $a, b \in K$. As we assumed the critical points of $f$ are distinct, we have $a \neq 0$. Without loss of generality, assume $h_{f}(a) \geq h_{f}(-a)$, and note this implies $h_{f}(a)>0$.

Proof of Theorem 1.1. Since $f$ is eventually stable, by Proposition 4.2 there exists some $N$ such that for any $\alpha \in f^{-N}(\beta)$, the pair $(f, \alpha)$ is stable. Let $\alpha_{1}, \ldots, \alpha_{3^{N}}$ be the elements of $f^{-N}(\beta)$. Note that $\left|f^{-N}(\beta)\right|=3^{N}$ because we have assumed that $\beta$ is not postcritical for $f$, so $f^{N}(x)-\beta$ has distinct roots in $\bar{K}$ for all $N \geq 1$.

We argue that $\alpha_{1}, \ldots, \alpha_{3^{N}}$ satisfy the assumptions of Proposition 9.1. As $\beta$ is not postcritical, no $\alpha_{i}$ lies in $\mathcal{O}_{f}(a)$ or $\mathcal{O}_{f}(-a)$, and all $\alpha_{i}$ are distinct. If $f^{\ell}\left(\alpha_{i}\right)=\alpha_{j}$ holds for $i \neq j$ and $\ell \geq 1$, then $f^{n}\left(\alpha_{i}\right)=f^{n-\ell}\left(\alpha_{j}\right)=\beta$ for some $m<n$ and $f^{\ell}(\beta)=\beta$, contradicting eventual stability of $(f, \beta)$ by Proposition 4.2. Likewise, if $b=0$ and $f^{\ell}\left(-\alpha_{i}\right)=\alpha_{j}$, then $-f^{\ell}\left(\alpha_{i}\right)=\alpha_{j}$ because $f$ is odd, whence $f^{n}\left(\alpha_{j}\right)=f^{n-\ell}\left(-f^{\ell}\left(\alpha_{i}\right)\right)=-f^{n}\left(\alpha_{i}\right)$, so $\beta=$ $-\beta$ and $\beta=0$. Then $f(\beta)=\beta$, again contradicting eventual stability. Thus Proposition 9.1 holds. For all sufficiently large $n$, there exist primes $\mathfrak{p}_{1}, \ldots, \mathfrak{p}_{3^{N}}$ of $K_{N}(\beta)$ satisfying conditions (a) through (c) of Proposition 6.7 for $\boldsymbol{\alpha}=\left(\alpha_{1}, \ldots, \alpha_{3^{N}}\right)$. Since $f^{n}(x)-\alpha_{i}$ is irreducible over $K\left(\alpha_{i}\right)$, condition (d) holds as well. Thus, Proposition 6.7 implies that $\left|\operatorname{Gal}\left(K_{n}(\boldsymbol{\alpha}) / K_{n-1}(\boldsymbol{\alpha})\right)\right|=6^{3^{N+n-1}}$. Since $K_{n}(\boldsymbol{\alpha})=K_{N+n}(\beta)$ and $K_{n-1}(\boldsymbol{\alpha})=K_{N+n-1}(\beta)$, we thus have

$$
\frac{\left.\mid G_{N+n}(\beta)\right) \mid}{\left.\mid G_{N+n-1}(\beta)\right)}=\operatorname{Gal}\left(K_{N+n}(\beta) / K_{N+n-1}(\beta)\right) \mid=\frac{\left|\operatorname{Aut}\left(T_{N+n}\right)\right|}{\left|\operatorname{Aut}\left(T_{N+n-1}\right)\right|}
$$

for all sufficiently large $n$, which means that the index of $G_{\infty}(f, \beta)$ in $\operatorname{Aut}\left(T_{\infty}\right)$ must be finite, as desired.

\section{The STUnted TREe}

Let $f \in K(x)$ and $\beta \in \mathbb{P}^{1}(\bar{K})$. We define the stunted tree $T_{n}^{s}(\beta)$ inductively as follows in terms of its $n$th level $\mathcal{L}_{n}(\beta)$. For $n=0$, set $T_{0}^{s}(\beta)=$ $\mathcal{L}_{0}(\beta)=\{\beta\}$. For $n \geq 1$, define $\mathcal{L}_{n}(\beta)$ by

$$
\mathcal{L}_{n}(\beta)=\left\{z \in \mathbb{P}^{1}(\bar{K}): f^{n}(z)=\beta\right\} \backslash T_{n-1}^{s}(\beta) .
$$

In other words, $\mathcal{L}_{n}(\beta)$ consists of the "strict" inverse images of $\beta$ under $f^{n}$, the $z \in f^{-n}(\beta)$ that did not appear as inverse images of $\beta$ under $f^{m}$ for 
some $m<n$. Then define

$$
T_{n}^{s}(\beta)=\bigcup_{i=0}^{n} \mathcal{L}_{i}(\beta),
$$

noting that this union is disjoint by definition. The idea behind this construction is that $T_{n}^{s}(\beta)$ is the "actual" tree of preimages of $\beta$ as opposed to the "idealized" tree of preimages $T_{n}(\beta)$. Each point in $T_{n}^{s}(\beta)$ occurs precisely once, as points are not repeated in the tree due to periodicity or to account for ramification at critical points. In particular, $\mathcal{L}_{n}(\beta)$ is a set and not a multiset. For example, see Figure 3 and compare with Figure 2 from Section 2 ,
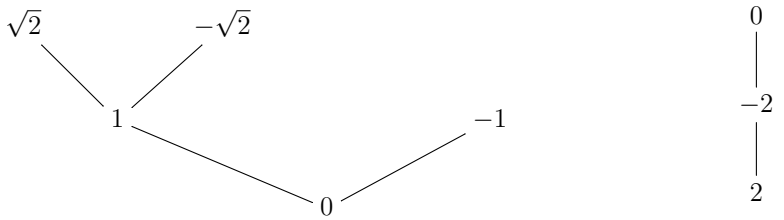

Figure $3 . T_{2}^{s}(0)$ for $x^{2}-1$ and $T_{2}^{s}(2)$ for $x^{2}-2$

Let $T_{\infty}^{s}(\beta)$ be the direct limit of the $T_{n}^{s}(\beta)$. As before, the group $G_{n}(\beta)$ acts faithfully on the tree $T_{n}^{s}(\beta)$, and this action commutes with the action of $f$ on $T_{n}^{s}(\beta) \backslash \beta$, so there is an injection $G_{n}(\beta) \hookrightarrow \operatorname{Aut}\left(T_{n}^{s}(\beta)\right)$ for each $n$. Taking inverse limits, there is an injection $G_{\infty}(\beta) \hookrightarrow \operatorname{Aut}\left(T_{\infty}^{s}(\beta)\right)$.

For $n \in \mathbb{N} \cup\{\infty\}$, there is a surjective morphism of rooted trees $T_{n}(\beta) \rightarrow$ $T_{n}^{s}(\beta)$ where the fiber over each $z \in T_{n}^{s}(\beta)$ is the set of vertices in $T_{n}(\beta)$ labeled by $z$. Thus $T_{n}^{s}(\beta)$ is a quotient tree of $T_{n}(\beta)$. The quotient morphism is an isomorphism if and only if no element of $\mathbb{P}^{1}(\bar{K})$ occurs more than once in $T_{\infty}$; this holds in turn if and only if $\beta$ is not periodic and not postcritical for $f$. Also note that the morphism is Galois-equivariant. So there are injections $\operatorname{Aut}\left(T_{n}^{s}(\beta)\right) \hookrightarrow \operatorname{Aut}\left(T_{n}(\beta)\right)$ for each $n \in \mathbb{N} \cup\{\infty\}$, and the image of the arboreal Galois representation $\rho_{f, \beta}: \operatorname{Gal}(\bar{K} / K) \rightarrow \operatorname{Aut}\left(T_{\infty}\right)$ is contained in the subgroup $\operatorname{Aut}\left(T_{\infty}^{s}(\beta)\right)$. If $\left[\operatorname{Aut}\left(T_{\infty}\right): \operatorname{Aut}\left(T_{\infty}^{s}(\beta)\right)\right]=\infty$, then $G_{\infty}(\beta)$ cannot have finite index in $\operatorname{Aut}\left(T_{\infty}\right)$, but it may still be possible that $G_{\infty}(\beta)$ has finite index in $\operatorname{Aut}\left(T_{\infty}^{s}(\beta)\right)$. Thus we have a variant of our original finite index problem, which does not appear to have been studied before.

Question 10.1. When is $\left[\operatorname{Aut}\left(T_{\infty}^{s}(\beta)\right): G_{\infty}(\beta)\right]<\infty$ ?

If $\beta$ is periodic or postcritical, then Question 10.1 may have a positive answer even though Question 2.1 cannot.

We define a variant of eventual stability that can hold even when $\beta$ is periodic or postcritical for $f$.

Definition 10.2. We say $(f, \beta)$ is tree-stable if there exists $n$ such that, for each $\alpha \in \mathcal{L}_{n}(\beta)$, the pair $(f, \alpha)$ is stable. 
If the pair $(f, \beta)$ is eventually stable, then it is also tree-stable by Proposition 4.2. Of course, the converse fails in general.

In Theorem 10.3 we give a nearly complete answer to Question 10.1 for cubic polynomials. We prove a set of necessary and sufficient conditions for $G_{\infty}(\beta)$ to have finite index in $\operatorname{Aut}\left(T_{\infty}^{s}(\beta)\right)$ when $K$ is a function field, provided that we exclude the possibility that $f$ is odd and $\beta=0$. If $K$ is a number field, the proof is conditional on $a b c$ and Vojta's conjecture just as in Theorem 1.1 .

The basic strategy to prove a finite index result in $\operatorname{Aut}\left(T_{\infty}^{s}(\beta)\right)$ is the same as for $\operatorname{Aut}\left(T_{\infty}\right)$. That is, we show that $\operatorname{Gal}\left(K_{n}(\beta) / K_{n-1}(\beta)\right)$ is maximal for all large $n$, which in this case means

$$
\operatorname{Gal}\left(K_{n}(\beta) / K_{n-1}(\beta)\right) \cong \operatorname{Aut}\left(T_{n}^{s}(\beta) / T_{n-1}^{s}(\beta)\right),
$$

where $\operatorname{Aut}\left(T_{n}^{s}(\beta) / T_{n-1}^{s}(\beta)\right)$ is the set of all automorphisms of $T_{n}^{s}(\beta)$ that act trivially on $T_{n-1}^{s}(\beta)$. Then finite index in $\operatorname{Aut}\left(T_{\infty}^{s}(\beta)\right)$ follows easily from the profinite structure of $G_{\infty}(\beta)$.

There is one more issue that arises in this context. If $f$ is odd and there is a $\gamma$ such that both $\gamma$ and $-\gamma$ are in $T_{\infty}^{s}(\beta)$, then $-\beta \in T_{\infty}^{s}(\beta)$ so there is some $\ell$ such that $f^{\ell}(-\beta)=\beta$. This means that $f^{2 \ell}(\beta)=-f^{\ell}(\beta)=\beta$ so $\beta$ is in a periodic cycle that is mapped to itself by $\sigma: x \mapsto-x$. Hence the entire tree $T_{\infty}^{s}(\beta)$ must be stable under $\sigma: x \mapsto-x$. This forces $\left[\operatorname{Aut}\left(T_{\infty}^{s}(\beta)\right.\right.$ : $\left.G_{\infty}(\beta)\right]=\infty$ (see Proposition 11.3 for details in a more general context). This is similar to the dynamical analogs of complex multiplication described by Jones [Jon13, Section 3.4].

Theorem 10.3. Let $K$ be a number field or a function field of characteristic 0 and transcendence degree one over an algebraic extension of $\mathbb{Q}$. Let $f(x)=$ $x^{3}-3 a^{2} x+b \in K[x]$ and let $\beta \in K$. If $K$ is a number field, assume the abc conjecture for $K$ and Vojta's conjecture for blowups of $\mathbb{P}^{1} \times \mathbb{P}^{1}$. If $K$ is a function field, assume that $f$ is not isotrivial. If $f$ is odd, suppose furthermore that there is no $\gamma$ such that both $\gamma$ and $-\gamma$ are in $T_{\infty}^{s}(\beta)$.

The following are equivalent:

(1) The pair $(f, \beta)$ is tree-stable, $f$ is not PCF, $f$ has distinct finite critical points $a,-a$, and we have $f^{n}(a) \neq f^{n}(-a)$ for all $n \geq 1$.

(2) The group $G_{\infty}(\beta)$ has finite index in $\operatorname{Aut}\left(T_{\infty}^{s}(\beta)\right)$.

Proof. Assume that $\beta$ is either periodic or postcritical for $f$, as otherwise the theorem follows immediately from Theorem 1.1 .

The necessity of the the first three conditions in (1) is follows from their necessity in Theorem 1.1. In particular, $f^{-1}(\beta) \neq\{\beta\}$ (since if it was then $f(x)=x^{3}$, which is post-critically finite), so we may choose a $\gamma$ that is not periodic or post-critical such that $f^{n}(\gamma)=\beta$ for some $n$. If $G_{\infty}(\beta)$ has finite index in $\operatorname{Aut}\left(T_{\infty}^{s}(\beta)\right)$, then certainly $G_{\infty}(\gamma)$ has finite index in $\operatorname{Aut}\left(T_{\infty}(\gamma)\right)$, but as seen in Proposition 3.2, that can only happen if $f$ is not PCF, $f$ has distinct finite critical points $\gamma_{1}, \gamma_{2}$, and $f^{n}\left(\gamma_{1}\right) \neq f^{n}\left(\gamma_{2}\right)$ for all $n \geq 1$. 
We turn to the problem of showing the listed conditions are sufficient. We may choose an $N$ such that none of the elements in $\mathcal{L}_{N}(\beta)$ are periodic are post-critical or periodic and such that $f^{n}(x)-\gamma$ is irreducible for all $\gamma \in \mathcal{L}_{N}(\beta)$.

Let $\left\{\alpha_{1}, \ldots, \alpha_{t}\right\}$ denote the distinct elements of $\mathcal{L}_{N}(\beta)$. Then $f^{N}\left(\alpha_{i}\right)=\beta$ for all $\alpha_{i}$ and $f^{m}\left(\alpha_{i}\right) \neq \beta$ for all $m<N$. If $f^{\ell}\left(\alpha_{i}\right)=\alpha_{j}$ for $\ell>0$, then $f^{N-\ell}\left(\alpha_{j}\right)=\beta$, a contradiction. Thus $\alpha_{i} \notin \mathcal{O}_{f}\left(\alpha_{j}\right)$ for $i \neq j$. Since no $\alpha_{i}$ is in $\mathcal{O}_{f}(a)$ or $\mathcal{O}_{f}(-a)$, we thus see that if $b \neq 0$, then $\alpha_{1}, \ldots, \alpha_{t}$ satisfy the conditions of Proposition 9.1. If $b=0$, we see that if $f^{\ell}\left(-\alpha_{j}\right)=\alpha_{i}$ for some $\ell>0$ then $\alpha_{j}$ and $-\alpha_{j}$ are both in $T_{\infty}^{s}(\beta)$, a contradiction. Thus, the conditions of Proposition 9.1 are also met in this case.

Letting $\boldsymbol{\alpha}=\left(\alpha_{1}, \ldots, \alpha_{t}\right)$ and applying Proposition 6.7, as in the proof of Theorem 1.1, we then see that for all sufficiently large $n$, we have

$$
\frac{\left|G_{N+n}(\beta)\right|}{\left|G_{N+n-1}(\beta)\right|}=\frac{\left|\operatorname{Aut}\left(T_{N+n}^{s}(\beta)\right)\right|}{\left|\operatorname{Aut}\left(T_{N+n-1}^{s}(\beta)\right)\right|},
$$

and our proof is complete.

\section{The multitree}

Let $f \in K(x)$ with $\operatorname{deg} f \geq 2$. Throughout the paper we have studied the Galois theory of the fields generated by taking preimages of one point $\beta$ under $f$. In this section, we simultaneously consider a collection of $s$ (not necessarily disjoint) trees rooted at distinct points $\beta_{1}, \ldots, \beta_{s} \in \mathbb{P}^{1}(\bar{K})$. To ease notation, set $\mathbf{B}=\left\{\beta_{1}, \ldots, \beta_{s}\right\}$. Recall the definition of $\mathcal{L}_{n}\left(\beta_{j}\right)$ from Section 10 as the $n$th level of a stunted tree rooted at $\beta_{j}$. Define

$$
\mathcal{M}_{n}(\mathbf{B})=\bigcup_{i=0}^{n} \bigcup_{j=1}^{s} \mathcal{L}_{n}\left(\beta_{j}\right)
$$

and

$$
G_{n}(\mathbf{B})=\operatorname{Gal}\left(K\left(\mathcal{M}_{n}(\mathbf{B})\right) / K(\mathbf{B})\right) .
$$

We refer to $\mathcal{M}_{n}(\mathbf{B})$ as a multitree. See Figure 4 for an example. As usual,
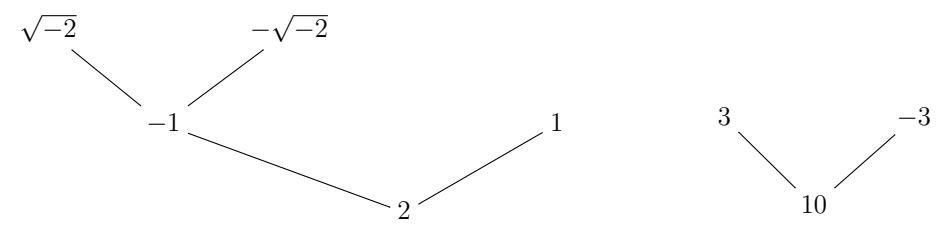

Figure $4 . \mathcal{M}_{1}(-1,2,10)$ for $x^{2}+1$

define $\mathcal{M}_{\infty}(\mathbf{B})$ to be the direct limit of the $\mathcal{M}_{n}(\mathbf{B})$ and $G_{\infty}(\mathbf{B})$ to be the inverse limit of the $G_{n}(\mathbf{B})$ as $n \rightarrow \infty$. For each $n, G_{n}(\mathbf{B})$ acts faithfully on $\mathcal{M}_{n}(\mathbf{B})$ in the usual way. So there are injections $G_{n}(\mathbf{B}) \hookrightarrow \operatorname{Aut}\left(\mathcal{M}_{n}(\mathbf{B})\right)$, 
and thus an injection $G_{\infty}(\mathbf{B}) \hookrightarrow \operatorname{Aut}\left(\mathcal{M}_{\infty}(\mathbf{B})\right)$, where an automorphism of the multitree must fix each root. Once again we have a finite index question:

Question 11.1. When is $\left[\operatorname{Aut}\left(\mathcal{M}_{\infty}(\mathbf{B})\right): G_{\infty}(\mathbf{B})\right]<\infty$ ?

We partially answer this question for cubic polynomials in Theorem 11.2 , which is a multitree version of Theorem 10.3 . As in Theorem 10.3 , we exclude certain cases arising from odd polynomials (and our proof is conditional on $a b c$ and Vojta's conjecture when $K$ is a number field). First we make some remarks about the structure of $\operatorname{Aut}\left(\mathcal{M}_{\infty}(\mathbf{B})\right)$.

Each stunted tree $T_{\infty}^{s}\left(\beta_{i}\right)$ injects into $\mathcal{M}_{\infty}(\mathbf{B})$, and the root of $T_{\infty}^{s}\left(\beta_{i}\right)$ maps to a root of $\mathcal{M}_{\infty}(\mathbf{B})$. If no $\beta_{i}$ is in the forward orbit of any other $\beta_{j}$, then $\mathcal{M}_{\infty}$ is precisely the disjoint union of the rooted trees $T_{\infty}^{s}\left(\beta_{i}\right)$ for $1 \leq i \leq s$ (this is known as a forest). In general, there is a root-preserving surjective morphism of graphs

$$
\bigsqcup_{i=1}^{s} T_{\infty}^{s}\left(\beta_{i}\right) \rightarrow \mathcal{M}_{\infty}(\mathbf{B})
$$

where the fiber over each $z \in \mathcal{M}_{\infty}(\mathbf{B})$ is the set of all vertices in the various trees labeled by $z$. This morphism is an isomorphism if and only if the trees $T_{\infty}^{s}\left(\beta_{i}\right)$ are disjoint. If the trees intersect, then we can describe $\mathcal{M}_{\infty}(\mathbf{B})$ as follows: partition $\mathbf{B}$ into classes based on membership in the same grand orbit, where grand orbits of $f$ in $\bar{K}$ are equivalence classes of the relation $\sim$ defined by $y \sim z$ if there exist $n, m \geq 0$ such that $f^{n}(y)=f^{m}(z)$. From each class, choose an element $\beta$ that is "farthest forward" in its grand orbit, that is, such that either $\beta$ is periodic or $f^{n}(\beta) \notin \mathbf{B}$ for all $n>0$. Let $\mathbf{A}=\left\{\gamma_{1}, \ldots, \gamma_{w}\right\}$ be the subset of $\mathbf{B}$ consisting of the chosen class representatives. Now we have the equality of unrooted graphs

$$
\mathcal{M}_{\infty}(\mathbf{B})=\mathcal{M}_{\infty}(\mathbf{A})=\bigsqcup_{i=1}^{w} T_{\infty}^{s}\left(\gamma_{i}\right),
$$

but the points $\beta_{i}$ are also marked as roots within each tree $T_{\infty}^{s}\left(\gamma_{i}\right)$, and are not moved by any element of $\operatorname{Aut}\left(\mathcal{M}_{\infty}(\mathbf{B})\right)$.

We describe the automorphism group of this disjoint union of trees. Let $A_{i}$ be the subgroup of $\operatorname{Aut}\left(T_{\infty}^{s}\left(\gamma_{i}\right)\right)$ that acts as the identity on the elements of $\mathbf{B}$ that lie in $T_{\infty}^{s}\left(\gamma_{i}\right)$. It is easy to see that $A_{i}$ has finite index in $\operatorname{Aut}\left(T_{\infty}^{s}\left(\gamma_{i}\right)\right)$. The group $A_{i}$ sits as a normal subgroup in Aut $\left(\bigsqcup_{i=1}^{w} T_{\infty}^{s}\left(\gamma_{i}\right)\right)$, and any $g \in \operatorname{Aut}\left(\bigsqcup_{i=1}^{w} T_{\infty}^{s}\left(\gamma_{i}\right)\right) \backslash \prod_{i=1}^{w} \operatorname{Aut}\left(T_{\infty}^{s}\left(\gamma_{i}\right)\right)$ must move some basepoint $\gamma_{i}$, i.e. $g\left(\gamma_{i}\right) \neq \gamma_{i}$ for some $i$. On the other hand, specifying a permutation of the set $\mathbf{A}$ and an automorphism of each stunted tree completely determines an automorphism of the disjoint union. Therefore

$$
\operatorname{Aut}\left(\mathcal{M}_{\infty}(\mathbf{B})\right) \cong \prod_{i=1}^{w} A_{i} \rtimes H
$$

for some subgroup $H$ of the symmetric group $\operatorname{Sym}(\mathbf{A}) \cong S_{w}$. More precisely, $H$ consists of the permutations in $S_{w}$ that preserve the partition of $\mathbf{A}$ defined 
by grouping together those basepoints $\gamma_{i}$ such that $A_{i}$ is isomorphic. As in the case of Theorem 10.3 , there are some added complications when $f$ is odd.

Theorem 11.2. Let $K$ be a number field or a function field of characteristic 0 and transcendence degree one over an algebraic extension of $\mathbb{Q}$. Let $f(x)=$ $x^{3}-3 a^{2} x+b \in K[x]$ with $\operatorname{deg} f=3$ and let $\beta_{1}, \ldots, \beta_{s} \in K$ be distinct. If $K$ is a number field, assume the abc conjecture for $K$ and Vojta's conjecture for blowups of $\mathbb{P}^{1} \times \mathbb{P}^{1}$. If $K$ is a function field, assume that $f$ is not isotrivial. If $f$ is an odd polynomial, assume furthermore that there is no $\gamma$ such that both $\gamma$ and $-\gamma$ are in $\mathcal{M}_{\infty}(\mathbf{B})$.

The following are equivalent:

(1) Each pair $\left(f, \beta_{i}\right)$ is tree-stable for $1 \leq i \leq s, f$ is not PCF, $f$ has distinct finite critical points $a,-a$, and $f^{n}(a) \neq f^{n}(-a)$ for all $n \geq 1$.

(2) The group $G_{\infty}(\mathbf{B})$ has finite index in $\operatorname{Aut}\left(\mathcal{M}_{\infty}(\mathbf{B})\right)$.

Proof. Let $\mathbf{A}=\left\{\gamma_{1}, \ldots, \gamma_{w}\right\}$ be a set of grand orbit representatives for $\mathbf{B}$ as in the discussion before the theorem.

If any one of the conditions fails, then by Theorem 10.3 , one of the groups $G_{\infty}\left(\beta_{i}\right)$ has infinite index in $\operatorname{Aut}\left(T_{\infty}^{s}\left(\beta_{i}\right)\right)$. As in the proof of Theorem 10.3 , this forces $G_{\infty}\left(\beta_{i}\right)$ to have infinite index in $\operatorname{Aut}\left(T_{\infty}^{s}\left(\gamma_{j}\right)\right.$ ) (where $\gamma_{j}$ is the chosen grand orbit representative for $\beta_{i}$ ) and thus infinite index in $A_{j}$. From the discussion of the structure of $\operatorname{Aut}\left(\mathcal{M}_{\infty}(\mathbf{B})\right)$ before the statement of the theorem, it follows easily that $G_{\infty}(\mathbf{B})$ has infinite index in $\operatorname{Aut}\left(\mathcal{M}_{\infty}(\mathbf{B})\right)$.

Now assume that all of the conditions hold. Take $N$ to be large enough so that $\mathbf{B} \subseteq \mathcal{M}_{N}(\mathbf{A})$ and that, for $1 \leq i \leq w$, each $\alpha \in \mathcal{L}_{N}\left(\gamma_{i}\right)$ is not periodic and not postcritical and has the property that $f^{n}(x)-\alpha$ is irreducible over $K(\alpha)$ for all $n$. Then, in particular, for every positive integer $n$ and every $\alpha \in \mathcal{L}_{N}\left(\gamma_{i}\right)$, the tree $T_{\infty}^{s}(\alpha)$ is the full $d$-ary tree $T_{\infty}(\alpha)$. Therefore

$$
K\left(\mathcal{M}_{N+n}(\mathbf{A})\right)=K\left(\mathcal{M}_{N}(\mathbf{A})\right)\left(\bigsqcup_{i=1}^{w} \bigsqcup_{z \in \mathcal{L}_{n}\left(\alpha_{i}\right)} T_{n}(z)\right)
$$

By our choice of $N$, we have $K\left(\mathcal{M}_{n}(\mathbf{B})\right) \subseteq K\left(\mathcal{M}_{N+n}(\mathbf{A})\right)$; thus $K\left(\mathcal{M}_{\infty}(\mathbf{A})\right)=$ $K\left(\mathcal{M}_{\infty}(\mathbf{B})\right)$ and $G_{\infty}(\mathbf{B})=\operatorname{Gal}\left(K\left(\mathcal{M}_{\infty}(\mathbf{A})\right) / K(\mathbf{B})\right)$.

We now argue exactly as in the proof of Theorem 10.3 . We write

$$
\bigcup_{i=1}^{w} \mathcal{L}_{N}\left(\gamma_{i}\right)=\left\{\alpha_{1}, \ldots, \alpha_{t}\right\}
$$

We cannot have $f^{\ell}\left(\alpha_{i}\right)=\alpha_{j}$ for $\ell>0$ and $i \neq j$ since $\alpha_{i}, \alpha_{j}$ are either in different grand orbits or are strict $N$-th inverse images of the same $\gamma \in \mathbf{A}$. If $f$ is odd, then there is no $\ell$ such that $f^{\ell}\left(-\alpha_{j}\right)=\alpha_{i}$ since otherwise we would have that $\alpha_{j}$ and $-\alpha_{j}$ are both in $\mathcal{M}_{\infty}(\mathbf{B})$. 
Letting $\boldsymbol{\alpha}=\left(\alpha_{1}, \ldots, \alpha_{t}\right)$ and applying Proposition 6.7, as in the proof of Theorem 1.1, we then see that for all sufficiently large $n$, we have

$$
\frac{\left|\operatorname{Gal}\left(K_{n}(\boldsymbol{\alpha})\right)\right|}{\left|\operatorname{Gal}\left(K_{n-1}(\boldsymbol{\alpha})\right)\right|}=\frac{\left|\operatorname{Aut}\left(\bigsqcup_{i=1}^{w} T_{N+n}^{s}\left(\gamma_{i}\right)\right)\right|}{\left|\operatorname{Aut}\left(\bigsqcup_{i=1}^{w} T_{N+n-1}^{s}\left(\gamma_{i}\right)\right)\right|},
$$

and our proof is complete, exactly as in the proof of Theorem 10.3 .

We also have the partial converse.

Proposition 11.3. With notation as above, suppose that $f(x)=x^{3}-3 a^{2} x$ (i.e., $f$ is odd) with $a \neq 0$ and that there exists $\gamma \in \bar{K}$ such that both $\gamma$ and $-\gamma$ are in $\mathcal{M}_{\infty}(\mathbf{B})$. Then $\left.\left[\operatorname{Aut}\left(\mathcal{M}_{\infty}(\mathbf{B})\right): G_{\infty}(\mathbf{B})\right)\right]=\infty$.

Proof. We may choose $\alpha,-\alpha$ such that $f^{n}(\alpha)=\gamma$ and neither $\alpha$ nor $-\alpha$ is periodic or post-critical (note that this implies in particular that $\alpha \neq 0$ ), Since $\operatorname{Gal}(\bar{K} / K(\alpha))$ has finite index in $\operatorname{Gal}(\bar{K} / K)$, we may suppose that $\alpha,-\alpha \in K$. Then the trees $T_{\infty}(\alpha)$ and $T_{\infty}(-\alpha)$ are both stable under the action of $\operatorname{Gal}(\bar{K} / K)$, and the map $\sigma: x \mapsto-x$ transposes $T_{\infty}(\alpha)$ and $T_{\infty}(-\alpha)$. Hence, any element of $\operatorname{Gal}(\bar{K} / K)$ that acts trivially on $T_{\infty}(\alpha)$ must act trivially on $T_{\infty}(-\alpha)$ as well. This means that the image of $\operatorname{Gal}(\bar{K} / K)$ in $\operatorname{Aut}\left(T_{\infty}(\alpha)\right) \times \operatorname{Aut}\left(T_{\infty}(-\alpha)\right.$ ) (induced by the action of $\operatorname{Gal}(\bar{K} / K)$ on $\left.\mathcal{M}_{\infty}(\mathbf{B})\right)$ cannot have finite index in $\operatorname{Aut}\left(T_{\infty}(\alpha)\right) \times \operatorname{Aut}\left(T_{\infty}(-\alpha)\right)$. Since $\operatorname{Aut}\left(\mathcal{M}_{\infty}(\mathbf{B})\right)$ contains $\operatorname{Aut}\left(T_{\infty}(\alpha)\right) \times \operatorname{Aut}\left(T_{\infty}(-\alpha)\right)$ as a subgroup, we must therefore have $\left.\left[\operatorname{Aut}\left(\mathcal{M}_{\infty}(\mathbf{B})\right): G_{\infty}(\mathbf{B})\right)\right]=\infty$, as desired.

\section{The ISOTRIVIAL CASE}

In this section, we treat the case where $f$ is isotrivial. Recall that we say that a rational function $\phi$ over a function field $K$ with field of constants $k$ is isotrivial if there exists $\sigma \in \bar{K}(x)$ of degree one such that $\sigma \phi \sigma^{-1} \in \bar{k}(x)$. Note that since one can always choose a map that sends any given point to the point at infinity, when $f$ is a polynomial this is equivalent to saying there is a $\sigma \in \bar{K}(x)$ of degree one such that $\sigma \phi \sigma^{-1} \in \bar{k}[x]$. We first the case where $f$ is a cubic with coefficients in $k$ and $\beta$ is transcendental over $k$.

Proposition 12.1. Let $k$ be any field of characteristic 0 , and let $f$ be a cubic polynomial in $k[x]$ such that $f$ is not $P C F, f$ has distinct finite critical points $\gamma_{1}, \gamma_{2}$, and $f^{n}\left(\gamma_{1}\right) \neq f^{n}\left(\gamma_{2}\right)$ for all $n \geq 1$. Suppose that $\beta$ is transcendental over $k$; let $K=k(\beta)$. Then $G_{\infty}(f, \beta)=\operatorname{Aut}\left(T_{\infty}\right)$.

Proof. After change of variables, we may write $f(x)=x^{3}-3 a^{2} x+b$. Since there is no $n$ such that $f^{n}(a)=f^{n}(-a)$ and $\mathrm{f}$ is not post-critically finite, we may assume that $a$ is not preperiodic and that there are no $i, j$ with $i \leq j$ such that $f^{i}(-a)=f^{j}(a)$. The polynomial $f^{n}(x)-\beta$ is easily seen to be irreducible over $k(\beta)$; for example, this follows immediately by looking at its Newton polygon at the place at infinity for $k(\beta)$. Arguing as in JKMT16, 
Section 3] and the proof of [BT18, Theorem 5.1], we see that for any $n$, the prime $\left(\beta-f^{n}(a)\right)$ in $K=k(\beta)$ satisfies Condition $R$ at $\beta$ for $n$. Then, as in the proof of Theorem 1.1, we have that $\operatorname{Gal}\left(K_{n}(\beta) / K_{n-1}(\beta)\right) \cong S_{3}^{3^{n-1}}$. Hence, by induction, $\left|G_{n}(f, \beta)\right|=\left|\operatorname{Aut}\left(T_{n}\right)\right|$ for all $n$.

The following is now an immediate consequence of Proposition 12.1 and Lemma 3.2 .

Corollary 12.2. Let $k$ be any algebraically closed field of characteristic 0 , let $K$ be a function field of transcendence degree 1 over $k$, and let $f$ be a cubic polynomial in $K[x]$ such that $f$ is not PCF, $f$ has distinct finite critical points $\gamma_{1}, \gamma_{2}$, and $f^{n}\left(\gamma_{1}\right) \neq f^{n}\left(\gamma_{2}\right)$ for all $n \geq 1$. Suppose that there exists $\sigma \in \bar{K}(x)$ of degree one such that $\sigma f \sigma^{-1} \in k(x)$. If $\sigma(\beta) \notin k$, then $G_{\infty}(f, \beta)$ has finite index in $\operatorname{Aut}\left(T_{\infty}\right)$.

We have a much more general result in the situation where the automorphism sending the coefficients of a rational function into the field of constants also sends $\beta$ into the field of constants.

Theorem 12.3. Let $k$ be any algebraically closed field, and let $K$ be any function field of transcendence degree 1 over $k$. Suppose that $\phi$ is a rational function in $K(x)$ such that there is a $\sigma \in \bar{K}(x)$ of degree one such that $\sigma f \sigma^{-1} \in k(x)$. Then, for any $\beta$ such that $\sigma(\beta) \in k$, the group $G_{\infty}(\phi, \beta)$ must be finite.

Proof. We argue as in [BT18, Theorem 5.1]. For any $n$ and any $\alpha$ such that $f^{n}(\alpha)=\beta$, we have $\sigma f^{n} \sigma^{-1} \sigma(\alpha)=\sigma(\beta) \in k$, so $\sigma(\alpha) \in k$, since $k$ is algebraically closed. Thus, $\alpha=\sigma^{-1}(z)$ for some $z \in k$. Since there is a finite extension $K^{\prime}$ of $K$ such that $\sigma \in K^{\prime}(x)$, we see that there is a finite extension $K^{\prime}$ of $K$ such that for any $\alpha$ and $n$ such that $f^{n}(\alpha)=\beta$, we have $\alpha \in K^{\prime}$, and our proof is complete.

Since Proposition 3.3 holds for isotrivial maps, we now have the following analog of Theorem 1.1 for isotrivial maps.

Theorem 12.4. Let $f$ be a cubic polynomial in $K[x]$, where $K$ is a function field of transcendence degree 1 over an algebraically closed field $k$ of characteristic 0. Let $\beta \in K$. Suppose there exists $\sigma \in \bar{K}[x]$ of degree one such that $\sigma f \sigma^{-1} \in k[x]$.

The following are equivalent:

(1) We have $\sigma(\beta) \notin k, f$ is not PCF, $f$ has distinct finite critical points $\gamma_{1}, \gamma_{2}$, and $f^{n}\left(\gamma_{1}\right) \neq f^{n}\left(\gamma_{2}\right)$ for all $n \geq 1$.

(2) The group $G_{\infty}(f, \beta)$ has finite index in $\operatorname{Aut}\left(T_{\infty}\right)$.

\section{REFERENCES}

[BCZ03] Y. Bugeaud, P. Corvaja, and U. Zannier, An upper bound for the G.C.D. of $a^{n}-1$ and $b^{n}-1$, Math. Z. 243 (2003), no. 1, 79-84. 
$\left[\mathrm{BFH}^{+} 16\right]$ R. L. Benedetto, X. Faber, B. Hutz, J. Juul, and Y. Yasufuku, A large arboreal Galois representation for a cubic postcritically finite polynomial, available at arXiv:1612.03358, 2016.

[BG06] E. Bombieri and W. Gubler, Heights in Diophantine geometry, New Mathematical Monographs, vol. 4, Cambridge University Press, Cambridge, 2006.

[BGHT17] A. Bridy, D. Ghioca, L.-C. Hsia, and T. J. Tucker, Iterated galois groups of quadratic polynomials, in preparation, 2017.

[BGKT12] R. L. Benedetto, D. Ghioca, P. Kurlberg, and T. J. Tucker, A case of the dynamical Mordell-Lang conjecture, Math. Ann. 352 (2012), no. 1, 1-26, With an appendix by Umberto Zannier.

$\left[\mathrm{BIJ}^{+} 17\right] \quad$ A. Bridy, P. Ingram, R. Jones, J. Juul, A. Levy, M. Manes, S. RubinsteinSalzedo, and J. H. Silverman, Finite ramification for preimage fields of postcritically finite morphisms, available at arXiv:1511.00194, to appear in Math. Res. Lett., 2017.

[BJ07] N. Boston and R. Jones, Arboreal Galois representations, Geom. Dedicata 124 (2007), 27-35.

[BJ09] The image of an arboreal Galois representation, Pure Appl. Math. Q. 5 (2009), no. 1, 213-225.

[BT18] A. Bridy and T. J. Tucker, ABC implies a Zsigmondy principle for ramification, J. Number Theory 182 (2018), 296-310.

[Cre89] J. E. Cremona, On the Galois groups of the iterates of $x^{2}+1$, Mathematika 36 (1989), no. 2, 259-261 (1990).

[CS93] G. S. Call and J. H. Silverman, Canonical heights on varieties with morphisms, Compositio Math. 89 (1993), no. 2, 163-205.

[CZ05] P. Corvaja and U. Zannier, A lower bound for the height of a rational function at S-unit points, Monatsh. Math. 144 (2005), no. 3, 203-224.

[DLT17] J. Doyle, A. Levy, and T. J. Tucker, Quadratic polynomials over function fields are eventually stable, in preparation, 2017.

[FG16] C. Favre and T. Gauthier, Classification of special curves in the space of cubic polynomials, available at arXiv:1603.05126, to appear in Internat. Math. Res. Notices, 2016.

[FS96] B. Fein and M. Schacher, Properties of iterates and composites of polynomials, J. London Math. Soc. (2) 54 (1996), no. 3, 489-497.

[GKNY17] D. Ghioca, H. Krieger, K. D. Nguyen, and H. Ye, The dynamical André-Oort conjecture: unicritical polynomials, Duke Math. J. 166 (2017), no. 1, 1-25.

[GNT13] C. Gratton, K. Nguyen, and T. J. Tucker, ABC implies primitive prime divisors in arithmetic dynamics, Bull. Lond. Math. Soc. 45 (2013), no. 6, 11941208.

[GNT15] D. Ghioca, K. Nguyen, and T. J. Tucker, Portraits of preperiodic points for rational maps, Math. Proc. Cambridge Philos. Soc. 159 (2015), no. 1, 165-186.

[Gra98] A. Granville, ABC allows us to count squarefrees, Internat. Math. Res. Notices (1998), no. 19, 991-1009.

[Gre17] G. Grell, Profinite iterated monodromy groups for cubic polynomials, in preparation, 2017.

[GTZ08] D. Ghioca, T. J. Tucker, and M. E. Zieve, Intersections of polynomials orbits, and a dynamical Mordell-Lang conjecture, Invent. Math. 171 (2008), no. 2, 463-483.

[Gub08] W. Gubler, Equidistribution over function fields, Manuscripta Math. 127 (2008), no. 4, 485-510.

[GY16] D. Ghioca and H. Ye, The dynamical andre-oort conjecture for cubic polynomials, available at arXiv:1603.05303, to appear in Internat. Math. Res. Notices, 2016. 
[Hin13] W. Hindes, Points on elliptic curves parametrizing dynamical Galois groups, Acta Arith. 159 (2013), no. 2, 149-167.

[Hin15] The arithmetic of curves defined by iteration, Acta Arith. 169 (2015), no. $1,1-27$.

[HJM15] S. Hamblen, R. Jones, and K. Madhu, The density of primes in orbits of $z^{d}+c$, Int. Math. Res. Not. IMRN (2015), no. 7, 1924-1958.

[HS00] M. Hindry and J. H. Silverman, Diophantine geometry, Graduate Texts in Mathematics, vol. 201, Springer-Verlag, New York, 2000, An introduction.

[Hua17] K. Huang, Generalized greatest common divisors for the orbits under rational functions, available at arXiv:1702.03881, 2017.

[Ing13] P. Ingram, Arboreal Galois representations and uniformization of polynomial dynamics, Bull. Lond. Math. Soc. 45 (2013), no. 2, 301-308.

[IS09] P. Ingram and J. H. Silverman, Primitive divisors in arithmetic dynamics, Math. Proc. Cambridge Philos. Soc. 146 (2009), no. 2, 289-302.

[JKMT16] J. Juul, P. Kurlberg, K. Madhu, and T. J. Tucker, Wreath products and proportions of periodic points, Int. Math. Res. Not. IMRN (2016), no. 13, 3944-3969.

[JL16] R. Jones and A. Levy, Eventually stable rational functions, available at arXiv:1603.00673, to appear in Int. J. Number Theory., 2016.

[JM14] R. Jones and M. Manes, Galois theory of quadratic rational functions, Comment. Math. Helv. 89 (2014), no. 1, 173-213.

[Jon07] R. Jones, Iterated Galois towers, their associated martingales, and the p-adic Mandelbrot set, Compos. Math. 143 (2007), no. 5, 1108-1126.

[Jon08] - The density of prime divisors in the arithmetic dynamics of quadratic polynomials, J. Lond. Math. Soc. (2) 78 (2008), no. 2, 523-544.

[Jon13] , Galois representations from pre-image trees: an arboreal survey, Actes de la Conférence "Théorie des Nombres et Applications", Publ. Math. Besançon Algèbre Théorie Nr., vol. 2013, Presses Univ. Franche-Comté, Besançon, 2013, pp. 107-136.

[Jon15] _ Fixed-point-free elements of iterated monodromy groups, Trans. Amer. Math. Soc. 367 (2015), no. 3, 2023-2049.

[Juu16] J. Juul, Iterates of generic polynomials and generic rational functions, available at arXiv:1410.3814, to appear in Trans. Amer. Math. Soc., 2016.

[Kri13] H. Krieger, Primitive prime divisors in the critical orbit of $z^{d}+c$, Int. Math. Res. Not. IMRN (2013), no. 23, 5498-5525.

[Loo16] N. Looper, Dynamical galois groups of trinomials and odoni's conjecture, available at arXiv:1609.03398v2, 14 pages, 2016.

[MS94] P. Morton and J. H. Silverman, Rational periodic points of rational functions, Internat. Math. Res. Notices (1994), no. 2, 97-110.

[MS14] A. Medvedev and T. Scanlon, Invariant varieties for polynomial dynamical systems, Ann. of Math. (2) 179 (2014), no. 1, 81-177.

[Odo85] R. W. K. Odoni, The Galois theory of iterates and composites of polynomials, Proc. London Math. Soc. (3) 51 (1985), no. 3, 385-414.

[Odo88] _ Realising wreath products of cyclic groups as Galois groups, Mathematika 35 (1988), no. 1, 101-113.

[Pin13a] R. Pink, Finiteness and liftability of postcritically finite morphisms in arbitrary characteristic, available at arXiv:1305.2841, 2013.

[Pin13b]_, Profinite iterated monodromy groups arising from quadratic morphisms with infinite postcritical orbits, available at arXiv:1309.5804, 2013.

[Pin13c] _ Profinite iterated monodromy groups arising from quadratic polynomials, available at arXiv:1307.5678, 2013.

[Rez79] B. Reznick, When is the iterate of a formal power series odd?, J. Austral. Math. Soc. Ser. A 28 (1979), no. 1, 62-66. 
[Rit20] J. F. Ritt, On the iteration of rational functions, Trans. Amer. Math. Soc. 21 (1920), no. 3, 348-356.

[Rit23] $\quad$ Permutable rational functions, Trans. Amer. Math. Soc. 25 (1923), no. 3, 399-448.

[Ser72] J.-P. Serre, Propriétés galoisiennes des points d'ordre fini des courbes elliptiques, Invent. Math. 15 (1972), no. 4, 259-331.

[Sil05] J. H. Silverman, Generalized greatest common divisors, divisibility sequences, and Vojta's conjecture for blowups, Monatsh. Math. 145 (2005), no. 4, 333350 .

[Sil07] _ The arithmetic of dynamical systems, Graduate Texts in Mathematics, vol. 241, Springer, New York, 2007.

[Sto92] M. Stoll, Galois groups over $\mathbf{Q}$ of some iterated polynomials, Arch. Math. (Basel) 59 (1992), no. 3, 239-244.

[Voj87] P. Vojta, Diophantine approximations and value distribution theory, Lecture Notes in Mathematics, vol. 1239, Springer-Verlag, Berlin, 1987.

[Xie15] J. Xie, The Dynamical Mordell-Lang Conjecture for polynomial endomorphisms of the affine plane, available at arXiv:1503.00773, 2015.

[Yua08] X. Yuan, Big line bundles over arithmetic varieties, Invent. Math. 173 (2008), no. $3,603-649$.

Andrew Bridy, Department of Mathematics, Texas A\&M University, ColLEGE STATION, TX, 77843, USA

E-mail address: andrewbridy@tamu.edu

Thomas J. Tucker, Department of Mathematics, University of Rochester, ROCHESTER, NY, 14620, USA

E-mail address: thomas.tucker@rochester.edu 